\title{
The Q-loops of the Human Multidrug Resistance Transporter ABCB1 are Necessary to Couple Drug Binding to the ATP Catalytic Cycle
}

Joseph K. Zolnerciks ${ }^{1, a}$, Begum G. Akkaya ${ }^{1, a}$, Marjolein Snippe ${ }^{1}$, Peter Chiba ${ }^{2}$, Anna Seelig ${ }^{3}$ and Kenneth J. Linton ${ }^{1}$

\footnotetext{
${ }^{1}$ Blizard Institute, Barts and The London School of Medicine and Dentistry, Queen Mary, University of London, London UK.

${ }^{2}$ Institute of Medical Chemistry, Medical University of Vienna, Vienna, Austria.

${ }^{3}$ Biophysical Chemistry Biozentrum, University of Basel, Basel, Switzerland.

${ }^{a}$ please consider as joint first authorship
}

Address correspondence to Professor Kenneth J. Linton, Centre for Cutaneous Research, Blizard Institute, Barts and The London School of Medicine and Dentistry, Queen Mary, University of London, 4 Newark Street, E1 2AT, UK. Tel. +44 (0)20 7882-8997; Fax +44 (0)20 7882-2200; Email: k.j.linton@qmul.ac.uk

Running title: Energy Transduction within an $\mathrm{ABC}$ transporter 


\section{Abbreviations}

$\mathrm{ABC}, \mathrm{ATP}$ binding cassette

ECAR, extracellular acidification rate

$\mathrm{MSH}$, membrane spanning helices

NBD, nucleotide binding domain

TMD, transmembrane domain 


\begin{abstract}
For a primary active pump like the human ATP-Binding-Cassette (ABC) transporter $\mathrm{ABCB} 1$, coupling of drug-binding by the two transmembrane domains (TMDs) to the ATP catalytic cycle of the two nucleotide-binding domains (NBDs) is fundamental to the transport mechanism but is poorly understood at the biochemical level. Structure data suggest that signals are transduced through intracellular loops of the TMDs that slot into grooves on the NBDs. At the base of these grooves is the Q-loop. We therefore mutated the eponymous glutamine in one or both NBD Q-loops and measured the impact on conformation and function using a conformation-sensitive antibody and fluorescent drugs, respectively. We show that the double mutant is trapped in the inward-open state which binds drug but cannot couple to the ATPase cycle. Our data also describe remarkable redundancy within the transport mechanism because single Q-loop mutants are functional for Bodipy-verapamil transport. This allowed us to elucidate transduction pathways from twin drug-binding cavities to the Q-loops using point mutations to favour one cavity over the other. Together, the data show that the Q-loop is the central flexion point where the aspect of the drug-binding cavities is coupled to the ATP catalytic cycle.
\end{abstract}

\title{
Key Terms
}

ABC transporter molecular mechanism

Drug efflux pump

MDR1 


\section{Introduction}

The human ATP Binding Cassette $(\mathrm{ABC})$ transporter $\mathrm{ABCB} 1$, formerly known as Pglycoprotein and MDR1, is an efflux pump of very broad solute specificity that includes lipid-signalling molecules and drugs of therapeutic value. Its function is known to cause clinical multidrug resistance (1). $\mathrm{ABCB} 1$ is a prototypical $\mathrm{ABC}$ transporter consisting of four domains in a single polypeptide (2): two nucleotide-binding domains (NBDs) form two composite ATP-binding pockets, and two transmembrane domains (TMDs) form the translocation pathway across the lipid bilayer and impart specificity by binding the transported drugs (3-5).

$\mathrm{X}$-ray structure models of five homologous $\mathrm{ABC}$ exporters are present in the protein database (drug efflux pumps from Mus musculus (Abcbla) (6), Staphylococcus aureus (Sav1866) (7) and Caenorhabditis elegans (CeAbcb1) (8); the lipid A exporter from various Gram negative bacteria (9); and a mitochondrial transporter of unknown function (ABCB10) from Homo sapiens (10)). The apo forms of four of these, Abcbla, CeAbcb1, MsbA from Vibrio cholera and $\mathrm{ABCB} 10$ crystallized in an inward-open conformation in which there is no direct intramolecular contact between the two NBDs (depicted in the left hand model of Figure 1A). Their TMD $\alpha$-helices are angled such that they describe an inverted ' $V$ ', forming an apex at the extracellular face of the membrane. The large internal cavity, open towards the inner leaflet of the membrane and the cytosol, is thought to form the solutebinding surface (Abcbla was also co-crystallized with cyclic peptide inhibitors bound in this cavity). The resolution of the Abcbla structure does not allow precise assignment of the amino acids involved in co-ordinating the bound drugs, but pharmacological labelling and modelling studies demonstrate the presence of two cavities at the TMD:TMD interface which are related by two-fold pseudo-symmetry and which can be separated by mutation (11-13). Both cavities are capable of binding verapamil, and the bundles of $\alpha$-helices formed by the TMDs as they cross the membrane ensures that amino acid residues from both TMDs line each cavity (Figure 1B) (12). In contrast, MsbA from Salmonella typhimurium and Sav1866 were crystallized in an inward-closed conformation (depicted in the central and right hand models of Figure 1A). The NBDs of these transporters are in close apposition and 
co-ordinate nucleotide or nucleotide analogues at their interface. In this inward-closed conformation the TMD $\alpha$-helices describe a regular ' $\mathrm{V}$ ' that exposes the putative solute binding sites extracellularly. Co-crystals of ABCB10 with nucleotide analogues were also obtained. These holo structures crystallised both in an inward-open state and in various partially-closed states suggesting that nucleotide by itself is insufficient to trigger formation of an inward-closed conformation for $\mathrm{ABCB} 10$ that is stable under crystallization conditions.

Taken together, the structure data suggest a bellows-like mechanism (Figure 1A) that conforms to the alternate access mode of action of membrane pumps first proposed by Jardetzky in 1966 (14). For ABCB1, drugs are reported to bind to the TMDs directly from the inner leaflet of the membrane. These would need to induce conformational change at the NBDs which allows the NBDs to interact directly and bind two molecules of ATP at their interface (the structure data for ABCB10 suggests that the NBDs may be poised with ATP already bound to the F1-core subdomains (see below) in the absence of drug). In ABCB1, nucleotide binding is reported to be sufficient to lower the affinity for drug and presumably also reconfigures the drug-binding cavities to release drug extracellularly $(15,16)$. The energy released from subsequent ATP hydrolysis is thought to be used to drive the exporter back to the inward-open conformation to re-expose the binding cavities to the inner leaflet of the membrane and restore their high affinity for drugs. This coupling of the aspect and affinity of the drug-binding sites of the TMDs to the closure of the NBD:NBD interface around the bound ATPs is fundamental to the transport cycle of ABC exporters but is poorly understood at the molecular mechanistic level. It is to the nature of the TMD:NBD interface that we need to look to understand it. The $\mathrm{ABC}$ exporter structure data (modelled for ABCB1 in Figures 1C and 1D) shows that the conserved Q-loop occupies a position at the base of a cleft in the top surface of the NBD into which a short helix formed by the TMD is docked. Each TMD of ABC exporters forms two such helices at the apices of two long intracellular loops. These two helices contact different NBDs allowing both TMDs to influence both NBDs even when the two NBDs are spatially separated. The TMDs of type I and type II ABC importers, epitomised by the maltose importer $\mathrm{MalEFGK}_{2}(17)$ and the vitamin $\mathrm{B} 12$ importer $\mathrm{BtuC}_{2} \mathrm{D}_{2}$ (18), respectively, form only one "coupling" helix which, despite the lack of primary sequence similarity, is structurally and positionally equivalent to 
the ABC exporter helix that fits into the cleft lined by the Q-loop (19-21). The Q-loop also links the F1-core and $\alpha$-helical subdomains of the NBDs (best illustrated in Figure 3). In the inward-closed conformation of the ABC exporters, the two ATPs are bound between the F1core of one NBD and the $\alpha$-helical subdomain of the second (Figure 1D). Higher resolution structural data obtained for soluble bacterial NBDs crystallized in the presence and absence of nucleotides suggested that flexion of the Q-loop may allow movement of the $\alpha$-helical subdomain with respect to the F1-core to control allosteric coupling between the two ATPbinding pockets (22-24). The Q-loop is therefore critically positioned to couple ligand binding by the TMDs to ATP binding at the NBDs in all ABC transporters.

We report here the first evidence of the crucial role played by the Q-loops in the intramolecular coupling mechanism of human ABCB1. Our approach was to mutate the eponymous glutamine in one, or both, NBD Q-loops and measure the impact on ABCB1 conformation and function. By combining these Q-loop mutants with further mutations in the TMDs that prevent occupancy of one or other drug-binding cavity we have also dissected the energy transduction pathways coupling the individual drug-binding cavities to the ATP catalytic cycle of the transporter.

\section{Materials and Methods}

Reagents. Dulbecco's modified eagle medium (DMEM), DMEM/F-12, fetal bovine serum (FBS), phosphate buffered saline (PBS), TrypLE Express, and Bodipy-verapamil were all purchased from Invitrogen (Carlsbad, CA). The detergent $n$-dodecyl- $\beta$-Dmaltoside was purchased from Merck Serono (Feltham, UK). Lipids were purchased from Avanti Polar Lipids (Alabaster, AL). All other general chemicals were from Sigma (Poole, UK).

Plasmids. Mutations were introduced into a plasmid encoding human ABCB1 with a Cterminal hexahistidine tag (pCIneo-wtABCB1-6His; described previously (25)) by sitedirected mutagenesis (QuikChange XL; Stratagene, La Jolla, CA) using the following oligonucleotides: 
Q132A, 5'-GGTTGCTGCTTACATCGCGGTTTCATTTTGGTGC-3';

Q132R, 5'-GGTTGCTGCTTACATTCGAGTTTCATTTTGGTGC-3';

Q475A, 5'-GGGAAATCATTGGTGTGGTGAGTGCTGAGCCTGTATTGTTTGCCACCACG-3';

Q773A, 5'-GGAATTATTTCTTTTATTACATTTTTCCTTGCGGGTTTCACATTTGGCAAAGCTGG-3';

Q773R, 5'-GGAATTATTTCTTTTATTACATTTTTCCTTCGAGGTTTCACATTTGGCAAAGCTGG-3';

Q1118A, 5'-GGGCATCGTGTCCGCGGAACCCATCCTGTTTG-3';

E556Q, 5'-CCCCAAGATCCTCCTGCTTGATCAGGCCACGTCAGCCTTGG-3';

E1201Q, 5'-CAGCCTCATATTTTGCTTCTTGATCAGGCCACGTCAGCTCTGGATAC-3'.

For expression in insect cells, the tagged wild-type and mutant $A B C B 1$ cDNAs were subcloned into the baculovirus transfer vector pBlueBAC4.5 (Invitrogen, Carlsbad, CA) using BamHI and Not I restriction sites at the 5'- and 3'-ends of the gene, respectively. The veracity of all plasmids was confirmed by DNA sequencing.

Expression in human cells. HEK293T cells were transfected-transiently using polyethyleneimine (PEI), as described previously (25). Equivalent expression levels of each mutant $\mathrm{ABCB} 1$ and wild-type protein were confirmed using saturating amounts of the ABCB1-specific primary antibody 4E3 (AbD Serotec, Oxford, UK) and Rphycoerythrin-conjugated goat anti-mouse secondary antibodies (Dako UK Ltd, Ely, UK), as described previously (26). UIC2-PE binding was carried out as described by the supplier (Immunotech, Beckman Coulter, CZ).

Drug transport assays. Each $\mathrm{ABCB} 1$ mutant was assessed for function using the fluorescent drug accumulation assay, described previously $(25,27)$. Briefly, HEK293T cells were transfected transiently with plasmids encoding wild-type or mutant ABCB1 and harvested 48 hours post-transfection. The live cells were incubated with 4E3 antibody, as described above, to label the surface ABCB1, and then with Bodipyverapamil $(0.8 \mu \mathrm{M})$ for $30 \mathrm{~min}$ at $37^{\circ} \mathrm{C}$ to assess transport activity. The fluorescence associated with cells of normal size and granularity was quantified using a FACScan flow cytometer. Flow cytometry data were acquired using CellQuest (BD Biosciences, San Jose, CA) and analysed using FlowJo (Tree Star, Ashland, OR). As each population of transfected cells contains both transfected (ABCB1-expressing) and untransfected (ABCB1-negative) cells distinguished on the basis of $4 \mathrm{E} 3$ antibody binding, the 
untransfected subpopulation was used as an internal control for measuring Bodipyverapamil accumulation. ABCB1 transport activity was assessed as the fold difference in drug accumulation between the ABCB1-expressing and non-expressing cell populations within each experiment.

Confocal microscopy. Primary antibody 4E3 (AbD Serotec, Oxford, UK) was conjugated to Alexa633 dye using the Mix-n-Stain (Biotium, Hayward, CA, USA) according to the manufacturer's instructions. Transiently-transfected HEK293T cells were split, 24h post-transfection, onto glass-chamber slides (Lab-Tek, Thermo Fisher Scientific, Waltham, Massachusetts, USA). 48hrs post-transfection, the live cells were washed 3x in DMEM/F12 medium then incubated with the Alexa633-conjugated 4E3 antibody (1/100 dilution in DMEM/F12 medium) plus Bodipy-verapamil $(0.8 \mu \mathrm{M})$ for $30 \mathrm{~min}$ at $37^{\circ} \mathrm{C}, 5 \% \mathrm{CO}_{2}$. The cells were washed $3 \mathrm{x}$ then imaged using a Zeiss LSM510 inverted confocal laser scanning microscope. Images were obtained using a planapochromat 63x oil objective with a numerical aperture of 1.4. Bodipy was excited using the Argon laser (488nm laser line) and Alexa633 using the HeNe laser (633nm). Bodipy emission was detected using the HFT UV/488/543/633 main dichroic beam splitter in combination with a band pass filter (BP 505-530) and Alexa633 was detected using a NFT 545 secondary beam splitter and a long pass filter (LP650). Multitrack mode allowed for crosstalk-free imaging of the two dyes.

Purification of ABCB1. Generation of recombinant baculovirus, growth and maintenance of High Five insect cells and the production of crude membrane fractions was as described elsewhere (28). ABCB1 was purified from crude membrane fractions using Ni-NTA resin (Qiagen) as described previously (29), with the following modifications. Initial binding of solubilised membrane protein to the resin was performed in the presence of $5 \mathrm{mM}$ imidazole; wash steps used $20 \mathrm{mM}, 30 \mathrm{mM}$ and $35 \mathrm{mM}$ imidazole. Purified protein was eluted from the resin in $120 \mathrm{mM}$ imidazole. To monitor purification, proteins were separated by $7.5 \%$ SDS-PAGE and visualized by colloidal blue staining. 


\section{Reconstitution of purified $\mathrm{ABCB} 1$ into proteoliposomes and measurement of}

ATPase activity. ABCB1 was reconstituted into liposomes as described previously (29).

To assess efficiency, the lipid was spiked with $\left[{ }^{3} \mathrm{H}\right]$-phosphatidylcholine and lipid and protein were equilibrated in a sucrose gradient by ultracentrifugation. Protein and lipid content of the fractions were analysed by SDS-PAGE and liquid scintillation counting, respectively, as detailed elsewhere (28).

\section{Cytosensor microphysiometer measurements of extracellular acidification rates} (ECAR). ECAR of live HEK293T cells were measured in an eight-channel Cytosensor microphysiometer (Molecular Devices, Menlo Park, CA), as described elsewhere (31-34). Briefly, $6 \times 10^{5}$ cells were seeded onto $12 \mathrm{~mm}$ diameter polycarbonate cell capsule cups in $1 \mathrm{ml}$ cell culture medium and incubated for $6 \mathrm{~h}$ at $37^{\circ} \mathrm{C}$. On transfer to the Cytosensor, the culture medium was replaced by pumping low buffer-capacity flow medium (prepared from dry powder DMEM without sodium bicarbonate, but supplemented with $1 \mathrm{mM}$ sodium pyruvate to reduce basal ECAR values, and $2.6 \mathrm{~g} / \mathrm{L}$ sodium chloride to preserve osmolarity) through the flow chamber at a rate of $100 \mu 1 / \mathrm{min}$. The cells were left to equilibrate until a constant ECAR was reached (30-60 minutes) before switching the medium flowing over the cells to drug-containing medium. A measuring cycle lasted 2 minutes during which the flow was switched off for 20 seconds to measure acidification yielding one data point. Each stimulation cycle for a given drug concentration was repeated several times, after which the cells were washed with pure medium until the basal activity was recovered. All stimulations were reversible. Cells are primarily glycolytic in culture, hence, one molecule of glucose consumed is converted into two molecules of ATP and two molecules of lactic acid. The latter are secreted from the cell and quantified via ECAR measurements. As ATP is resynthesized on demand, the number of lactic acid molecules extruded directly corresponds to the number of ATP molecules consumed.

ECAR data were analysed using a two-site binding model based on uncompetitive inhibition, described previously $(31,35-37)$, in which drug activates $\mathrm{ABCB} 1$ at low concentrations and inhibits at high concentrations. 


$$
V_{s}=\frac{K_{1} K_{2} V_{0}+K_{2} V_{1} C_{s}+V_{2} C_{s}^{2}}{K_{1} K_{2}+K_{2} C_{s}+C_{s}{ }^{2}}
$$

$V_{s}$, ECAR as a function of the drug concentration $C_{s} ; V_{0}$, basal ECAR in the absence of drug; $V_{l}$, maximum ECAR assuming only activation; $V_{2}$, activity at infinite drug concentration; $K_{1}$, drug concentration giving half-maximum activation; $K_{2}$, drug concentration giving half-maximum reduction.

Statistical analyses. Student's two-tailed t-test was used to analyse differences between means. Differences among means were analysed using two-way ANOVA followed by pairwise Student-Newman-Keuls post hoc testing.

\section{Results}

\section{The Q-loops of the NBDs are essential for drug efflux but redundancy is built into} the molecular mechanism. Site-directed mutagenesis was used to introduce glutamineto-alanine mutations into NBD1 (NBD1-Q475A), NBD2 (NBD2-Q1118A), and into both NBDs of human ABCB1. As controls, mutations E556Q and E1201Q in the Walker B motifs of each NBD that render the transporter catalytically-inactive were also generated. HEK293T cells were transfected transiently with each of the plasmids and the expression and function of the mutant and wild-type transporters was analysed by two-wavelength flow cytometry. The non-inhibitory anti-ABCB1 antibody (4E3) which recognises an extracellular loop of the transporter was used at saturating concentrations with a red fluorescent secondary to determine the level of expression at the plasma membrane, and the green fluorescent drug derivative (Bodipy-verapamil) was used to assess transport activity. Figure 2A shows a representative dotplot for a population of cells transfected with pCIneo-wtABCB1-6His showing that cells expressing ABCB1 (upper left population) accumulate very little Bodipy-verapamil compared with non-transfected cells (lower right population) within the same sample. The fold difference in drug accumulation between the G1 and G2 gated cells provides a measure of the transport activity of $\mathrm{ABCB} 1$. None of the mutations introduced into $\mathrm{ABCB} 1$ had a significant 
effect on the level of protein expression in HEK293T cells as shown in the example histogram describing 4E3 staining (Figure 2B). Cell populations with equal surface expression of mutant and wild-type forms of $\mathrm{ABCB} 1$ could therefore be gated for analysis of transport function (analytical gates G1 and G2 in Figure 2, correspond to regions M1 and M2 in Figure 2B, respectively). Cells expressing the single NBD Q-loop mutants, NBD1-Q475A, or NBD2-Q1118A, accumulated only low levels of Bodipy-verapamil similar, but not identical to, those expressing wild-type ABCB1 (Figure 2C). The reproducible but subtle difference in Bodipy-verapamil transport activity by the single Qloop mutants did not reach statistical significance (shown in the first four columns of Figure 6). In contrast, cells expressing the double mutant Q475A/Q1118A exhibited no efflux activity, and rather surprisingly, and reproducibly, accumulated more Bodipyverapamil than mock-transfected cells or cells expressing the Walker B mutant E1201Q of $\mathrm{ABCB} 1$ (Figure 2C; the raw dotplot data also showed that Bodipy-verapamil accumulation increases linearly with increasing expression of Q475A/Q1118A (Supplementary Figure $1))$.

\section{The double Q-loop Q475A/Q1118A mutant is trapped in the inward-open}

conformation. The extracellular loops of human ABCB1 form a discontinuous epitope for the antibody UIC2, characterised by Igor Roninson's group $(38,39)$. UIC2 is sensitive to the conformation of $\mathrm{ABCB} 1$ and binds preferentially to ATP-free $\mathrm{ABCB} 1$, corresponding to the inward-open conformation shown in Figure 1A. Consequently, UIC2 binds more readily to wild-type ABCB1 than the Walker B mutant E1201Q (Figure 2D) in cells that express equal amounts of these proteins, because the Walker B mutant can bind but cannot hydrolyse ATP (40). The Walker B mutants therefore get trapped in the inward-closed conformation with a stable NBD:NBD interface around the bound ATP. UIC2 reproducibly binds most readily to the Q475A/Q1118A mutant suggesting that it adopts a conformation consistent with the inward-open state that would be expected to have a high affinity for UIC2. The single Q-loop mutants exhibit a phenotype for UIC2 binding that is indistinguishable from the wild-type transporter, reflecting the levels of functionality of these single mutants. 
The additional Bodipy-verapamil accumulated by cells that express the double Q-loop mutant prompted examination of the subcellular localisation of the accumulated drug. Confocal microscopy showed that cells expressing wild-type ABCB1 (stained with the non-inhibitory 4E3 conjugated to a red-fluorophore) accumulated no fluorescent drug (Figure 3, upper panels) because the wild-type transporter efficiently effluxes drug from the cells. Non-expressing cells included in the same field of view accumulated the green Bodipy-verapamil in intracellular compartments. In contrast, cells expressing the Q475A/Q1118A mutant accumulated Bodipy-verapamil both in intracellular compartments and the plasma membrane where it co-localises with the 4E3 staining (Figure 3, middle panels). Prior data shows that in the absence of ATP, ABCB1 adopts a conformation with a high affinity for drug (16). The microscopy data is therefore consistent with the UIC2-binding data and suggests that the Q475A/Q1118A adopts the inward-open conformation which, in the absence of the two Q-loop glutamines cannot form the NBD:NBD interface, provides additional drug-binding sites in the plasma membrane, and explains the increased accumulation of Bodipy-verapamil in these cells. In contrast, no Bodipy-verapamil was detected bound to the Walker B E1201Q mutant which becomes trapped in the inward-closed conformation. Cells expressing this mutant therefore accumulate drug only intracellularly (Figure 3, lower panels). This is also consistent with previously published data which shows that non-hydrolysable trinucleotides trap $\mathrm{ABCB} 1$ in a conformation with a low affinity for drug (16).

\section{The single Q-loop mutants retain the ability to hydrolyse ATP in live cells but not} post-purification. The drug export activity of the single Q-loop mutants was unexpected because equivalent mutations in mouse Abcb1 a were reported to reduce drug-stimulated ATPase activity by $>95 \%$ (41). In this earlier paper, the activity of the purified protein was characterised thoroughly in vitro, but drug transport was not analysed. Our observations of the human transporter, interpreted in light of this prior data, suggested that drug transport may have become uncoupled from ATPase activity in $\mathrm{ABCB} 1$. We therefore replicated the Abcb1 a experiments performed previously, with the human transporter. Wild-type and mutant forms of the human transporter were expressed in 
insect cells, solubilised from the membrane using n-dodecyl- $\beta$-D-maltoside (DDM), and purified by nickel-affinity chromatography (Figure 4A). ABCB1 is inactive in DDM at the concentrations needed for solubilisation therefore the purified protein was mixed with detergent-solubilised membrane lipids and $\mathrm{ABCB} 1$-liposomes were reconstituted by the slow removal of detergent.

The kinetics of drug-stimulated ATPase activity of wild-type and mutant ABCB1 fitted the Michaelis-Menten equation (Figure 4B). The low basal ATPase activity of the wildtype ABCB1 that was stimulated 11-fold by drug to a high $V_{\max }$ for ATP hydrolysis of 1.7 $\mu \mathrm{mol} / \mathrm{min} / \mathrm{mg}$ and a $K_{m}$ of $0.75 \mathrm{mM}$ (Table 1 ) compared favourably with previous reports of the catalytic activity of purified ABCB1, indicative of efficient reconstitution of the active transporter into proteoliposomes (42-44). The drug-stimulated ATPase activity of the single Q-loop mutants was reduced to $9.5 \%$ and $8.1 \%$ of the wild-type activity for the NBD1-Q475A and NBD2-Q1118A mutants, respectively, and the double mutant was inactive (the basal ATPase activities of these mutants were similarly affected; Figure 4B and $\mathrm{C}$ ). The observed difference was not due to the differential ability of the mutant proteins to form proteoliposomes as all three mutants reconstituted into the lipid with the same efficiency as the wild-type protein (Supplementary Figure 2). The data are largely consistent with the in vitro observations of Urbatsch et al., (41), effectively ruling out the possibility that the Q-loop mutants of mouse Abcb1a and human ABCB1 differ significantly.

These in vitro data suggested uncoupling of drug transport from ATPase activity in the single Q-loop mutants. However, to prove this unequivocally would require measurement of ATPase activity and drug transport in the same system. ABCB1 drug transport into proteoliposomes does not appear to be possible, most likely due to low protein:lipid ratios, the hydrophobicity, and the leakiness of large unilamellar liposomes to the transported drugs, so we therefore measured the ATPase activity of ABCB1 in intact human cells using extracellular acidification rates (ECAR) as a proxy. ECAR is noninvasive and measures the increase in lactate efflux from the cell which directly correlates to turnover of ATP upon drug stimulation in living cells (Figure 5A-E). The data best 
fitted a modified Michaelis-Menten equation producing bell-shaped curves, consistent with previous work which showed that a variety of different drugs stimulate ABCB1 at low concentrations but inhibit the transporter at high concentrations $(31,45)$.

Cells expressing wild-type $\mathrm{ABCB} 1$ exhibited a drug-stimulated acidification rate $\left(V_{l}\right.$ value) 1.34-fold above basal, whereas mock transfected cells had a negligible response ( $<1.05$-fold), indicating that the increase in ECAR was due to the expression, and ATP catalytic activity, of ABCB1 (Table 2). The $V_{l}$ values for cells expressing single mutants NBD1-Q475A and NBD2-Q1118A were 1.19-fold and 1.13-fold above basal rates, respectively, corresponding to $50 \%$ and $35 \%$ of the ATPase activity of wild-type $\mathrm{ABCB} 1$. No increase in ECAR in the presence of verapamil was detected in cells expressing the Q-loop double mutant Q475A/Q1118A. It seems likely therefore, that the lack of correlation with the in vitro data is due to inactivation of the single Q-loop mutants during purification. The simplest conclusion is that 9 out of every 10 transporter proteins reconstituted into proteoliposome preparations of single Q-loop mutants are inactive thus accounting for the reduction in both basal and drug-stimulated ATPase activity in these preparations. Together, these data show that the very low ATPase activity in vitro described in Table 1 and reported previously is an artefact due to the instability of the Q-loop mutants during purification.

\section{The Q-loops are necessary to couple the drug-binding sites to the ATP binding sites.} The two pseudo-symmetrical drug-binding cavities of $\mathrm{ABCB} 1$ can be manipulated independently by mutagenesis of residues Q132 (TMD1) and Q773 (TMD2) that line the cavities (Figure 1B, (12)). Replacement of the two TMD glutamines with arginines is reported to repel positively-charged drugs but have no effect on neutral drugs. Single mutants in which the glutamine in either TMD1 or TMD2 was replaced with arginine had intermediate phenotypes consistent with transport of the positively-charged drug only from the remaining wild-type drug-binding cavity (12). We introduced these glutamine to arginine mutations into $\mathrm{ABCB} 1$ and assessed their effect on Bodipy-verapamil export using two-colour flow cytometry. The TMD mutations had no effect on ABCB1 expression at the plasma membrane (Supplementary Figure 3). The single mutant TMD1- 
Q132R was able to efflux Bodipy-verapamil with 70\% efficiency of the wild-type protein while the TMD2-Q773R was fully active and indistinguishable from wild-type ABCB1 (grey bars in Figure 6 in which transport data are presented as the fold reduction in Bodipy-verapamil accumulation compared to mock-transfected cells). These data are consistent with prior observations which concluded that verapamil had a preference for binding to the cavity lined by Q132 (12). The double arginine mutant Q132R/Q773R, retains ability to export Bodipy-verapamil but it is significantly reduced to $42 \%$ ( $\mathrm{p}<0.001)$ of wild-type ABCB1 activity. This residual activity is likely due to the partial masking of the positive charge on verapamil by the Bodipy moiety such that electrostatic repulsion from Q132R or Q773R is incomplete. The catalytically-inactive Walker B mutants NBD1-E556Q and NBD2-E1201Q (46) were also tested for comparison and are unable to efflux Bodipy-verapamil.

To test whether each verapamil-binding cavity was coupled to the NBDs via a specific Qloop we combined the single drug-cavity mutants TMD1-Q132R and TMD2-Q773R with the NBD Q-loop mutants NBD1-Q475A and NBD2-Q1118A and compared their transport activity (striped bars in Figure 6) with that of the drug cavity mutants and also the single and double Q-loop mutants (white bars; note that the double Q-loop mutant has a fold-difference that is less than 1 because it provides additional binding sites for Bodipy-verapamil in the membrane). The drug-cavity mutant TMD1-Q132R combined synergistically with NBD1-Q475A to significantly reduce Bodipy-verapamil export activity to $25 \%$ of wild-type activity, but in combination with NBD2-Q1118A the transporter retained the full level of activity of each single mutant (at $85 \%$ of the wildtype activity this was not significantly different from the Q132R mutant or the Q1118A mutant). This shows that the wild-type Q773-lined verapamil-binding cavity of the Q132R mutant is dedicated to, and only requires, the NBD1 Q-loop to couple to the ATP catalytic cycle. In contrast, TMD2-Q773R combined synergistically with both NBD1Q475A and NBD2-Q1118A to reduce Bodipy-verapamil export activity to $22 \%$ and $34 \%$ of the wild-type activity, respectively. This shows that the wild-type Q132-lined verapamil-binding cavity of these mutants is coupled to, and requires, the Q-loops of both NBDs to trigger efflux. 


\section{Discussion}

\section{The Q-loop is the flexion point where the aspect and affinity of the drug-binding cavities couple to the ATP catalytic cycle.}

The Q-loops are essential to the molecular mechanism of ABCB1, because the double Qloop mutant is trapped in an inward-open conformation that retains affinity for Bodipyverapamil but cannot trigger the transport cycle and therefore cannot efflux Bodipyverapamil. In contrast, the single Q-loop mutants retain wild-type levels of Bodipyverapamil transport activity, demonstrating a surprising level of redundancy in the mechanism that is not observed for Walker B mutants of ABC exporters ((47) and data herein) or in ABC importers (48). The high level of Bodipy-verapamil efflux activity of the single Q-loop mutants in live cells allowed the role of the Q-loop in the coupling mechanism of ABCB1 to be investigated. The single Q-loop mutants combined with TMD mutants, Q132R in TMD1 or Q773R in TMD2, which line the two verapamilbinding cavities (12), showed that Bodipy-verapamil bound to the cavity lined by TMD2Q773 triggers conformation change to the inward-closed state via the conduit of the Qloop in NBD1. In reciprocal experiments, in which the transporter preferentially engaged drug via the Q132-lined cavity (by introduction of the Q773R mutation) both Q-loops were required to efficiently couple efflux of the bound Bodipy-verapamil to the ATP catalytic cycle. Together, these data place the Q-loops at the centre of the molecular mechanism where they control the coupling of the alternate access cycle of the TMDs with the ATP catalytic cycle of the NBDs.

We envisage the role of the Q-loop in the transport cycle of an ABC exporter as follows. The drug-binding cavities of ABCB1 in the inward-open state are exposed to the inner leaflet of the membrane and its NBDs are completely separated as observed in crystals of apo MsbA, Abcb1a, CeAbcb1 and ABCB10, in a conformation made permissible by the direct contact of each TMD with both NBDs. ATP may well be bound by the F1-core of each NBD but the $\alpha$-helical subdomain is not engaged (this is the case for ABCB10, and also for the maltose importer $\mathrm{MalFGK}_{2}$ in the absence of maltose and its periplasmic 
binding protein MalE (49)). Drug accesses the inward-open ABCB1 from the inner leaflet of the membrane to occupy the cavities lined by Q132 or Q773. The interaction of drug with the TMDs triggers conformational change that is transmitted through one or both coupling helices (depending on which cavity is occupied and, most likely, on the chemistry of the drug/cavity interaction) to bring the two NBDs together. The coupling helix most likely flexes the Q-loop to allow the eponymous glutamine to hydrogen bond with the $\gamma$-phosphate of the ATP that is bound to the F1-core of the same domain (as observed in the high resolution structures of soluble bacterial NBDs (22-24)). This flexion also rotates the $\alpha$-helical subdomain forward to hydrogen bond with the second ATP bound to the F1-core of the apposed NBD. In the absence of both Q-loop glutamines $\mathrm{ABCB} 1$ cannot prosecute this conformational change. If only one Q-loop glutamine is intact, the transporter presumably locks one ATP into position but not the second. The full or partial closure of the NBD:NBD interface (in the wild type and single Q-loop mutants, respectively) causes a further conformational change in which the TMD helices are re-orientated to close the entrance gate and open the exit gate to release the bound drug to the extracellular milieu. The inward-closed conformation is most likely autocatalytic (MalK, the isolated NBDs of the maltose importer will hydrolyse ATP constitutively in vitro (50)). In ABCB1, hydrolysis of the ATP $\gamma$-phosphate likely releases the Q-loop and disengages the two NBDs to permit return to the inward-open conformation. We speculate that only the ATP locked into position by a hydrogen bond to its intradomain Q-loop is hydrolysed. This may explain the reduction by half of the verapamil-stimulated ATPase activity of cells expressing the single Q-loop mutants without diminution of the Bodipy-verapamil efflux activity. It would also explain the pronounced phenotype of the single Walker B mutants, because in this case both ATPs would be hydrogen bonded to the two Q-loops and both may therefore need to be hydrolysed to disengage the NBDs.

Acknowledgements. We are extremely grateful to Tasha Ritchie for her practical investigations and intellectual contribution to this manuscript. We also thank Matthias Zwick and Cinzia Esposito for assistance with Cytosensor experiments and valuable discussions. We are grateful to Thomas Stockner for use of his homology model of 
ABCB1. This work was supported by the Medical Research Council UK and a FEBS short-term fellowship to JKZ. BGA is funded by Queen Mary, University of London.

\section{References}

1. Gottesman, M. M. (2002) Mechanisms of cancer drug resistance. Annu Rev Med 53, 615-627

2. Higgins, C. F., Hiles, I. D., Salmond, G. P., Gill, D. R., Downie, J. A., Evans, I. J., Holland, I. B., Gray, L., Buckel, S. D., Bell, A. W., and et al. (1986) A family of related ATP-binding subunits coupled to many distinct biological processes in bacteria. Nature 323, 448-450

3. van Veen, H. W., Margolles, A., Muller, M., Higgins, C. F., and Konings, W. N. (2000) The homodimeric ATP-binding cassette transporter LmrA mediates multidrug transport by an alternating two-site (two-cylinder engine) mechanism. Embo J 19, 2503-2514

4. Shapiro, A. B., and Ling, V. (1997) Extraction of Hoechst 33342 from the cytoplasmic leaflet of the plasma membrane by P-glycoprotein. Eur J Biochem 250, 122-129

5. Li-Blatter, X., and Seelig, A. (2010) Exploring the P-glycoprotein binding cavity with polyoxyethylene alkyl ethers. Biophys $J$ 99, 3589-3598

6. Aller, S. G., Yu, J., Ward, A., Weng, Y., Chittaboina, S., Zhuo, R., Harrell, P. M., Trinh, Y. T., Zhang, Q., Urbatsch, I. L., and Chang, G. (2009) Structure of Pglycoprotein reveals a molecular basis for poly-specific drug binding. Science 323, 1718-1722

7. Dawson, R. J., and Locher, K. P. (2006) Structure of a bacterial multidrug ABC transporter. Nature 443, 180-185

8. Jin, M. S., Oldham, M. L., Zhang, Q., and Chen, J. (2012) Crystal structure of the multidrug transporter P-glycoprotein from Caenorhabditis elegans. Nature 490, 566-569

9. Ward, A., Reyes, C. L., Yu, J., Roth, C. B., and Chang, G. (2007) Flexibility in the ABC transporter MsbA: Alternating access with a twist. Proceedings of the National Academy of Sciences of the United States of America 104, 19005-19010

10. Shintre, C. A., Pike, A. C., Li, Q., Kim, J. I., Barr, A. J., Goubin, S., Shrestha, L., Yang, J., Berridge, G., Ross, J., Stansfeld, P. J., Sansom, M. S., Edwards, A. M., Bountra, C., Marsden, B. D., von Delft, F., Bullock, A. N., Gileadi, O., BurgessBrown, N. A., and Carpenter, E. P. (2013) Structures of ABCB10, a human ATPbinding cassette transporter in apo- and nucleotide-bound states. Proc Natl Acad Sci U S A 110, 9710-9715

11. Pleban, K., Kopp, S., Csaszar, E., Peer, M., Hrebicek, T., Rizzi, A., Ecker, G. F., and Chiba, P. (2005) P-glycoprotein substrate binding domains are located at the transmembrane domain/transmembrane domain interfaces: a combined photoaffinity labeling-protein homology modeling approach. Mol Pharmacol 67, 365-374 
12. Parveen, Z., Stockner, T., Bentele, C., Pferschy, S., Kraupp, M., Freissmuth, M., Ecker, G. F., and Chiba, P. (2011) Molecular dissection of dual pseudosymmetric solute translocation pathways in human P-glycoprotein. Mol Pharmacol 79, 443452

13. Donmez Cakil, Y., Khunweeraphong, N., Parveen, Z., Schmid, D., Artaker, M., Ecker, G. F., Sitte, H. H., Pusch, O., Stockner, T., and Chiba, P. (2013) Pore Exposed Tyrosine Residues of P-glycoprotein are Important Hydrogen Bonding Partners for Drugs. Molecular pharmacology

14. Jardetzky, O. (1966) Simple allosteric model for membrane pumps. Nature 211, 969-970

15. Higgins, C. F., and Linton, K. J. (2004) The ATP switch model for ABC transporters. Nat Struct Mol Biol 11, 918-926

16. Martin, C., Higgins, C. F., and Callaghan, R. (2001) The vinblastine binding site adopts high- and low-affinity conformations during a transport cycle of Pglycoprotein. Biochemistry 40, 15733-15742

17. Oldham, M. L., Khare, D., Quiocho, F. A., Davidson, A. L., and Chen, J. (2007) Crystal structure of a catalytic intermediate of the maltose transporter. Nature 450, 515-521

18. Locher, K. P., Lee, A. T., and Rees, D. C. (2002) The E. coli BtuCD structure: a framework for ABC transporter architecture and mechanism. Science 296, 10911098

19. Khare, D., Oldham, M. L., Orelle, C., Davidson, A. L., and Chen, J. (2009) Alternating access in maltose transporter mediated by rigid-body rotations. $\mathrm{Mol}$ Cell 33, 528-536

20. Kadaba, N. S., Kaiser, J. T., Johnson, E., Lee, A., and Rees, D. C. (2008) The high-affinity E. coli methionine $\mathrm{ABC}$ transporter: structure and allosteric regulation. Science 321, 250-253

21. Hollenstein, K., Frei, D. C., and Locher, K. P. (2007) Structure of an ABC transporter in complex with its binding protein. Nature 446, 213-216

22. Diederichs, K., Diez, J., Greller, G., Muller, C., Breed, J., Schnell, C., Vonrhein, C., Boos, W., and Welte, W. (2000) Crystal structure of MalK, the ATPase subunit of the trehalose/maltose $\mathrm{ABC}$ transporter of the archaeon Thermococcus litoralis. Embo J 19, 5951-5961

23. Gaudet, R., and Wiley, D. C. (2001) Structure of the ABC ATPase domain of human TAP1, the transporter associated with antigen processing. Embo J 20, 4964-4972

24. Verdon, G., Albers, S. V., Dijkstra, B. W., Driessen, A. J., and Thunnissen, A. M. (2003) Crystal structures of the ATPase subunit of the glucose ABC transporter from Sulfolobus solfataricus: nucleotide-free and nucleotide-bound conformations. Journal of molecular biology 330, 343-358

25. Blott, E. J., Higgins, C. F., and Linton, K. J. (1999) Cysteine-scanning mutagenesis provides no evidence for the extracellular accessibility of the nucleotide-binding domains of the multidrug resistance transporter Pglycoprotein. Embo J 18, 6800-6808 
26. Zolnerciks, J. K., Wooding, C., and Linton, K. J. (2007) Evidence for a Sav1866like architecture for the human multidrug transporter P-glycoprotein. Faseb J 21, 3937-3948

27. Dixon, P. H., Weerasekera, N., Linton, K. J., Donaldson, O., Chambers, J., Egginton, E., Weaver, J., Nelson-Piercy, C., de Swiet, M., Warnes, G., Elias, E., Higgins, C. F., Johnston, D. G., McCarthy, M. I., and Williamson, C. (2000) Heterozygous MDR3 missense mutation associated with intrahepatic cholestasis of pregnancy: evidence for a defect in protein trafficking. Hum Mol Genet $\mathbf{9}$, 1209-1217

28. Taylor, A. M., Storm, J., Soceneantu, L., Linton, K. J., Gabriel, M., Martin, C., Woodhouse, J., Blott, E., Higgins, C. F., and Callaghan, R. (2001) Detailed characterization of cysteine-less P-glycoprotein reveals subtle pharmacological differences in function from wild-type protein. Br J Pharmacol 134, 1609-1618

29. Ritchie, T. K., Grinkova, Y. V., Bayburt, T. H., Denisov, I. G., Zolnerciks, J. K., Atkins, W. M., and Sligar, S. G. (2009) Chapter 11 - Reconstitution of membrane proteins in phospholipid bilayer nanodiscs. Methods Enzymol 464, 211-231

30. Chifflet, S., Torriglia, A., Chiesa, R., and Tolosa, S. (1988) A method for the determination of inorganic phosphate in the presence of labile organic phosphate and high concentrations of protein: application to lens ATPases. Analytical biochemistry 168, 1-4

31. Landwojtowicz, E., Nervi, P., and Seelig, A. (2002) Real-time monitoring of Pglycoprotein activation in living cells. Biochemistry 41, 8050-8057

32. Nervi, P., Li-Blatter, X., Aanismaa, P., and Seelig, A. (2010) P-glycoprotein substrate transport assessed by comparing cellular and vesicular ATPase activity. Biochim Biophys Acta 1798, 515-525

33. Aanismaa, P., and Seelig, A. (2007) P-Glycoprotein kinetics measured in plasma membrane vesicles and living cells. Biochemistry 46, 3394-3404

34. McConnell, H. M., Owicki, J. C., Parce, J. W., Miller, D. L., Baxter, G. T., Wada, H. G., and Pitchford, S. (1992) The cytosensor microphysiometer: biological applications of silicon technology. Science 257, 1906-1912

35. Litman, T., Nielsen, D., Skovsgaard, T., Zeuthen, T., and Stein, W. D. (1997) ATPase activity of P-glycoprotein related to emergence of drug resistance in Ehrlich ascites tumor cell lines. Biochim Biophys Acta 1361, 147-158

36. Litman, T., Zeuthen, T., Skovsgaard, T., and Stein, W. D. (1997) Structureactivity relationships of P-glycoprotein interacting drugs: kinetic characterization of their effects on ATPase activity. Biochim Biophys Acta 1361, 159-168

37. Gatlik-Landwojtowicz, E., Aanismaa, P., and Seelig, A. (2006) Quantification and characterization of P-glycoprotein-substrate interactions. Biochemistry 45, 30203032

38. Mechetner, E. B., and Roninson, I. B. (1992) Efficient inhibition of Pglycoprotein-mediated multidrug resistance with a monoclonal antibody. Proc Natl Acad Sci U S A 89, 5824-5828

39. Zhou, Y., Gottesman, M. M., and Pastan, I. (1999) The extracellular loop between TM5 and TM6 of P-glycoprotein is required for reactivity with monoclonal antibody UIC2. Arch Biochem Biophys 367, 74-80 
40. Tombline, G., Bartholomew, L. A., Urbatsch, I. L., and Senior, A. E. (2004)

Combined mutation of catalytic glutamate residues in the two nucleotide binding domains of P-glycoprotein generates a conformation that binds ATP and ADP tightly. J Biol Chem 279, 31212-31220

41. Urbatsch, I. L., Gimi, K., Wilke-Mounts, S., and Senior, A. E. (2000) Investigation of the role of glutamine-471 and glutamine-1114 in the two catalytic sites of P-glycoprotein. Biochemistry 39, 11921-11927

42. Urbatsch, I. L., Wilke-Mounts, S., Gimi, K., and Senior, A. E. (2001) Purification and characterization of $\mathrm{N}$-glycosylation mutant mouse and human P-glycoproteins expressed in Pichia pastoris cells. Arch Biochem Biophys 388, 171-177

43. Kimura, Y., Kioka, N., Kato, H., Matsuo, M., and Ueda, K. (2007) Modulation of drug-stimulated ATPase activity of human MDR1/P-glycoprotein by cholesterol. Biochem J 401, 597-605

44. Rothnie, A., Theron, D., Soceneantu, L., Martin, C., Traikia, M., Berridge, G., Higgins, C. F., Devaux, P. F., and Callaghan, R. (2001) The importance of cholesterol in maintenance of P-glycoprotein activity and its membrane perturbing influence. Eur Biophys J 30, 430-442

45. al-Shawi, M. K., and Senior, A. E. (1993) Characterization of the adenosine triphosphatase activity of Chinese hamster P-glycoprotein. J Biol Chem 268, 4197-4206

46. Sauna, Z. E., Muller, M., Peng, X. H., and Ambudkar, S. V. (2002) Importance of the conserved Walker B glutamate residues, 556 and 1201, for the completion of the catalytic cycle of ATP hydrolysis by human P-glycoprotein (ABCB1). Biochemistry 41, 13989-14000

47. Urbatsch, I. L., Julien, M., Carrier, I., Rousseau, M. E., Cayrol, R., and Gros, P. (2000) Mutational analysis of conserved carboxylate residues in the nucleotide binding sites of P-glycoprotein. Biochemistry 39, 14138-14149

48. Tal, N., Ovcharenko, E., and Lewinson, O. (2013) A single intact ATPase site of the $\mathrm{ABC}$ transporter BtuCD drives 5\% transport activity yet supports full in vivo vitamin B12 utilization. Proc Natl Acad Sci U S A 110, 5434-5439

49. Orelle, C., Alvarez, F. J., Oldham, M. L., Orelle, A., Wiley, T. E., Chen, J., and Davidson, A. L. (2010) Dynamics of alpha-helical subdomain rotation in the intact maltose ATP-binding cassette transporter. Proc Natl Acad Sci U S A 107, 20293-20298

50. Walter, C., Wilken, S., and Schneider, E. (1992) Characterization of site-directed mutations in conserved domains of MalK, a bacterial member of the ATP-binding cassette (ABC) family [corrected]. FEBS Lett 303, 41-44

51. Stockner, T., de Vries, S. J., Bonvin, A. M., Ecker, G. F., and Chiba, P. (2009) Data-driven homology modelling of P-glycoprotein in the ATP-bound state indicates flexibility of the transmembrane domains. FEBS J 276, 964-972

52. Linton, K. J., and Higgins, C. F. (2007) Structure and function of ABC transporters: the ATP switch provides flexible control. Pflugers Arch 453, 555567

53. van der Does, C., and Tampe, R. (2004) How do ABC transporters drive transport? Biol Chem 385, 927-933 
54. Loo, T. W., Bartlett, M. C., and Clarke, D. M. (2006) Transmembrane segment 7 of human P-glycoprotein forms part of the drug-binding pocket. Biochem J 399, 351-359

55. Loo, T. W., and Clarke, D. M. (1997) Identification of residues in the drugbinding site of human P-glycoprotein using a thiol-reactive substrate. J Biol Chem 272, 31945-31948 
Table I. In vitro ATPase data for NBD1-Q475A, NBD2-Q1118A, and wildtype ABCB1.

Values shown are the average of at least 4 independent experiments \pm S.E.M. $* p<0.05$, *** $p<0.001$ versus wild-type ABCB1 using Student's two-tailed t-test. ${ }^{a}$ drug-stimulated $V_{\max }$ versus wild-type.

\begin{tabular}{|c|c|c|c|c|c|}
\hline & \multicolumn{2}{|c|}{ +Nicardipine $(50 \mu \mathrm{M})$} & \multicolumn{2}{|c|}{ - Nicardipine } & \multirow{2}{*}{$\begin{array}{c}\text { Relative } \\
\text { ATPase activity }^{\mathrm{a}} \\
(\%)\end{array}$} \\
\hline & $\boldsymbol{K}_{m}(\mathbf{m M})$ & $\begin{array}{c}V_{\max } \\
(\mathrm{nmol} \mathrm{Pi/min} / \mathrm{mg})\end{array}$ & $K_{m} .(\mathbf{m M})$ & $\begin{array}{c}V_{\max } \\
(\mathrm{nmol} \mathrm{Pi/min/mg)}\end{array}$ & \\
\hline Wild-type & $0.746 \pm 0.22$ & $1691 \pm 228$ & $0.327 \pm 0.49$ & $151 \pm 54$ & 100 \\
\hline Q475A & $2.37 \pm 1.64$ & $162 \pm 70^{* * *}$ & $0.284 \pm 0.19$ & $8.0 \pm 1.7 *$ & 9.5 \\
\hline Q1118A & $2.34 \pm 1.39$ & $138 \pm 51 * * *$ & $0.573 \pm 0.31$ & $8.2 \pm 1.7 *$ & 8.1 \\
\hline
\end{tabular}




\section{Table II. Cytosensor data for NBD1-Q475A, NBD2-Q1118A, Q475A/Q1118A and wild-type ABCB1.}

Values shown are the average of at least 3 independent experiments \pm S.E.M. $* * p<0.01$, *** $p<0.001$ versus wild-type ABCB1 using Student's two-tailed t-test. ND None detected.

\begin{tabular}{|c|c|c|c|c|c|}
\hline Mutant & $\boldsymbol{K}_{I}(\mu \mathbf{M})$ & $\begin{array}{c}V_{1} \\
\text { (fold increase) }\end{array}$ & $\begin{array}{c}V_{1} \\
(\% \text { wild-type })\end{array}$ & $\boldsymbol{K}_{2}(\mu \mathbf{M})$ & $\begin{array}{c}V_{2} \text { (Fold } \\
\text { increase) }\end{array}$ \\
\hline Wild-type & $0.56 \pm 0.03$ & $1.38 \pm 0.01$ & 100 & $23.05 \pm 3.44$ & $1.09 \pm 0.01$ \\
\hline Q475A & $0.91 \pm 0.10^{* * *}$ & $1.19 \pm 0.02 * * *$ & 50.2 & $26.54 \pm 4.84$ & $0.94 \pm 0.02 * * *$ \\
\hline Q1118A & $0.95 \pm 0.17 * *$ & $1.13 \pm 0.02 * * *$ & 35.6 & $84.81 \pm 30.36^{* * *}$ & $0.88 \pm 0.06^{* * *}$ \\
\hline Q475A/Q1118A & ND & ND & ND & ND & ND \\
\hline
\end{tabular}




\section{Figure legends}

Figure 1. Structural data for ABC exporters.

(A) A potential bellows-like mechanism of $\mathrm{ABC}$ exporters suggested by the structural studies. The inward-open conformation of Abcb1a is shown on the left (6). From the amino terminus: TMD1, yellow; NBD1, red; TMD2, blue; NBD2, cyan. The inwardclosed conformation of a homology model of human ABCB1 (51) is shown in the centre, and on the right rotated $90^{\circ}$ on the Y-axis. The inward-closed conformation is based on the Sav1866 structure (7) with ATP modelled into the NBDs. The mechanism $(15,52,53)$ is summarised in four steps (1) drug binds to the inward-open conformation from the inner leaflet of the bilayer. (2) This induces conformational change to allow the NBDs to close around two ATPs at the NBD:NBD interface. (3) The inward-closed state re-configures the drug-binding cavities such that they are now exposed extracellularly and simultaneously lowers the affinity for drug, which is released. (4) ATP hydrolysis, loss of phosphate (Pi) and ADP, restores the transporter to the inward-open state. (B) The verapamil binding cavities. The transmembrane $\alpha$-helices of the inward-open conformation of Abcb1a from above, showing the modelled side chains of residues (red sticks) implicated in verapamil binding $(54,55)$, the prospective verapamil binding cavities (dashed red lines), and the conserved glutamines (numbered as for human $\mathrm{ABCB} 1)$ that line the cavities and which when mutated to arginine inhibit binding to the adjacent cavity by electrostatic repulsion (spacefill orange and green). (C) Close-up view of coupling helix 2 of TMD1 (yellow) formed from the intracellular loop of transmembrane $\alpha$-helices 4 and 5 , as it contacts the cleft in NBD2 of the ABCB1 homology model. The model (viewed from the position of NBD1) shows the Q-loop (pink) linking the $\alpha$-helical subdomain (pale cyan) with the F1-core (cyan) of NBD2. The Q-loop also acts as conduit for interaction with the coupling helix. High-resolution structure data of bacterial NBDs suggest that the Q-loop glutamine (stick format) of ABCB1 will donate a hydrogen bond to the $\gamma$-phosphate of the ATP (stick format and coloured elementally) bound by the intradomain F1-core. Other TMD $\alpha$-helices have been removed for clarity. (D) Top-down view of the NBD:NBD interface of the ABCB1 homology model from the position of the TMDs. The F1-core (red) and $\alpha$-helical 
(salmon) subdomains of NBD1 are linked by the Q-loop (green). NBD2 is similarly shaded in cyan and pale cyan with the Q-loop in pink. The left hand image (cartoon format), and the right hand image (surface rendered) show the position of the Q-loops lining the base of the clefts that separate the F1-core and $\alpha$-helical subdomains.

Figure 2. Surface expression, conformational status, and effect on Bodipy-verapamil accumulation of mutant and wild-type ABCB1.

(A) A representative example of a two-wavelength flow cytometry dotplot of HEK293T cells transfected with pCIneo-wtABCB1-6His. Cells that express wild-type ABCB1 are labelled by the 4E3 anti-ABCB1 antibody and also extrude Bodipy-verapmil. The populations are pseudo-coloured blue to red to reflect low to high cell density. (B) A representative histogram showing total $\mathrm{ABCB} 1$ expression (4E3 binding) on the surface of transiently-transfected HEK293T cells. (C) A representative histogram showing the effect of the wild-type and mutant ABCB1 on Bodipy-verapamil accumulation. (D) A representative histogram showing binding of the conformation-sensitive ABCB1 antibody UIC2 to transiently-transfected HEK293T cells. Colour-coding is the same for all histograms.

Figure 3. Subcellular localisation of drug accumulation in HEK293T cells expressing wild type and mutant ABCB1.

ABCB1 was detected with non-inhibitory antibody 4E3 (red fluorescence; left hand panels), Bodipy-verapamil fluoresces green (centre panels). The right hand panels show the merged image. Wild-type ABCB 1 is highly active and exports Bodipy-verapamil from the cell such that these cells are red but not green (upper panels). Non-expressing cells accumulate Bodipy-verapamil intracellularly. The Q475A/Q1118A mutant binds Bodipyverapamil in the plasma membrane but cannot efflux it, thus drug accumulates in the plasma membrane of cells expressing the double Q-loop mutant ABCB1 and also in intracellular compartments (middle panels). In contrast, the catalytically inactive Walker B E1201Q mutant is trapped in the inward-closed conformation that has a low affinity for drug and these cells accumulate Bodipy-verapamil intracellularly but not in the plasma 
membrane (lower panels). Fields of view were selected to include a proportion of nontransfected cells in each panel for comparison.

Figure 4. Purification and ATPase activity of lipid-reconstituted wild-type and mutant human ABCB1.

(A) SDS-polyacrylamide gel stained with colloidal blue, showing total protein from each fraction. Lane 1 , crude membrane preparation ( $0.4 \%$ of total volume); lane 2 , solubilised membrane preparation ( $0.1 \%$ of total volume); lane 3 , unbound protein in the NiNTA resin flow-through $(0.1 \%$ of total volume); lanes $4-6$, washes $1-3$ (20mM imidazole; $2.5 \%$ of total volume); lane 7, wash 4 (30mM imidazole; $2.5 \%$ of total volume); lane 8 , wash 5 (35mM imidazole; $2.5 \%$ of total volume); lane 9, elution 0 (buffer $\mathrm{pH}$ change, $2 \mathrm{mM}$ imidazole; $2.5 \%$ of total volume); lanes $10-12$, elutions $1-3$ (120mM imidazole; $1.5 \%$ of total volume). (B) ATPase activity of wild-type and mutant ABCB1 in reconstituted proteoliposomes, as a function of increasing ATP concentration. Drug-stimulated ATPase activity was plotted as a function of ATP concentration using the Michaelis-Menten equation. (C) The single Q-loop mutants with a re-scaled y-axis to show the drugstimulation of their ATPase activities.

Figure 5. Drug-stimulated ATPase activity of wild-type and mutant ABCB1 measured in live cells.

(A) Extracellular acidification rates (ECARs) of HEK293T cells expressing wild-type (red), NBD1-Q475A (purple), or NBD2-Q1118A (orange) ABCB1, and mock-transfected cells (blue). Plots are shown as a function of time, with increasing verapamil concentration indicated above each experimental peak. (B-E) ECAR data was applied to a kinetic model based upon a modified Michaelis-Menten equation, described in the Materials and Methods. Representative plots from a single experiment, performed in duplicate with error bars indicating standard deviation, are shown using cells expressing (B) wild-type, (C) NBD1-Q475A, (D) NBD2-Q1118A, and (E) Q475A/Q1118A ABCB1.

Figure 6. Drug transport activity of wild-type and mutant ABCB1. 
HEK293T cells expressing ABCB1 were incubated with Bodipy-verapamil for 30min, before washing and FACS analysis. Values are calculated as the ratio of FL-1 (drug) fluorescence between $\mathrm{ABCB} 1$ expressing and non-expressing (untransfected cells) within the same sample. Each bar corresponds to the mutation(s) detailed in table below the graph. White bars indicate single/double NBD Q-loop mutants; grey, single/double TMD mutants; striped bars, double NBD/TMD mutants; black, wild-type; calalytically inactive Walker B mutants NBD1-E556Q and NBD2-E1201Q are indicated and serve as negative controls. Values shown are the average of at least 5 independent experiments \pm S.E.M. $\dagger$ cells expressing the Q475A/Q1118A mutant $\mathrm{ABCB} 1$ accumulate more Bodipy-verapamil than the untransfected cells giving a ratio of less than $1 . * p<0.05$, *** $p<0.001$, ns, not significant versus wild-type using one-way ANOVA with Student-Newman-Keuls posttest. 
Figure 1.

A

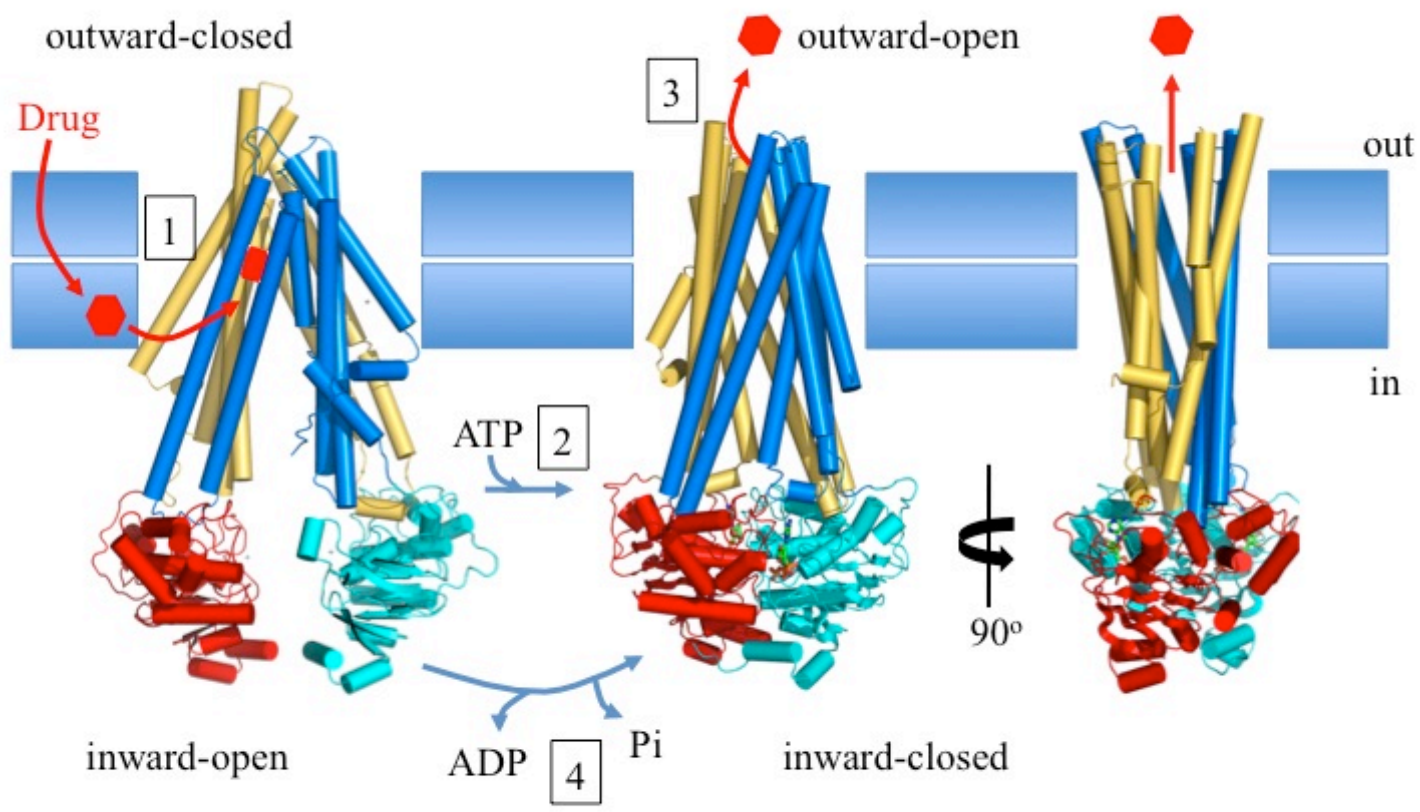

B

C
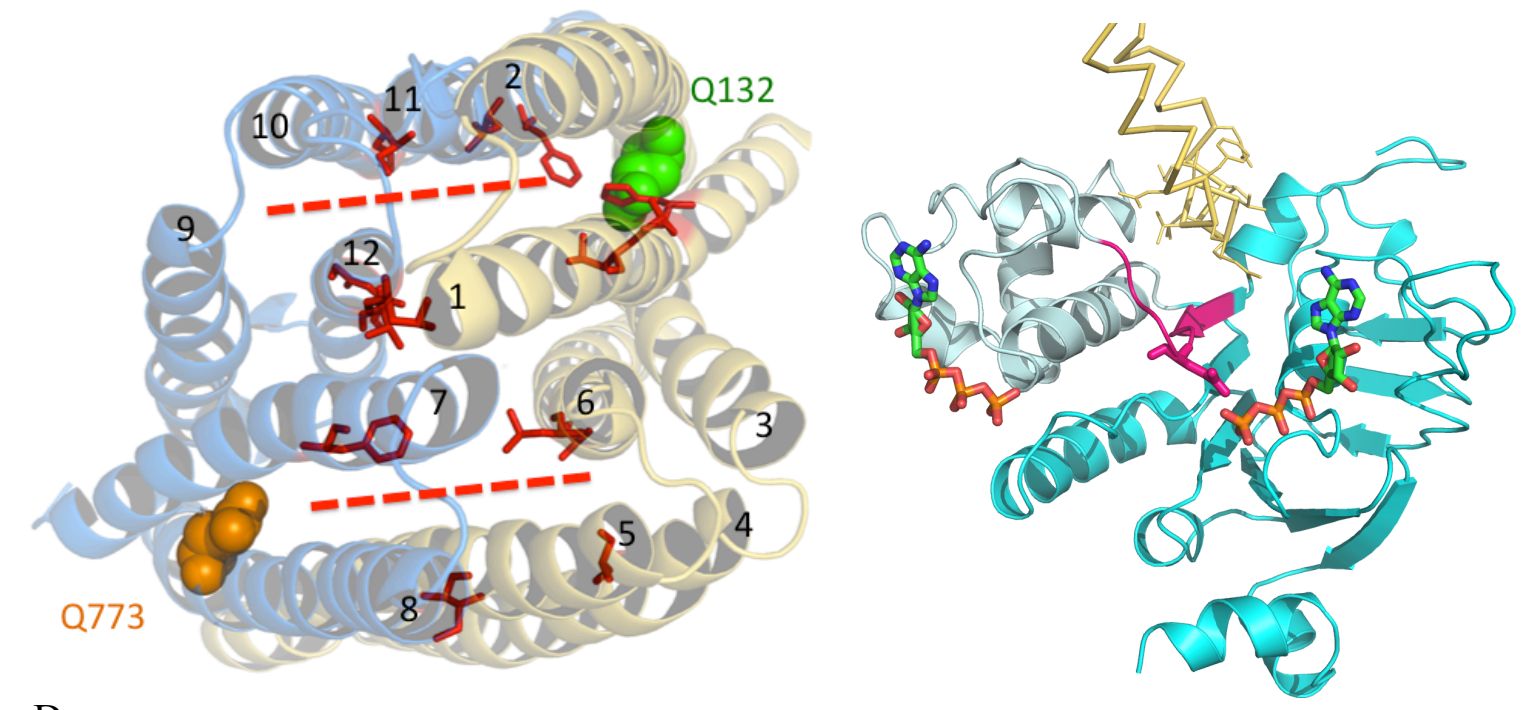

D
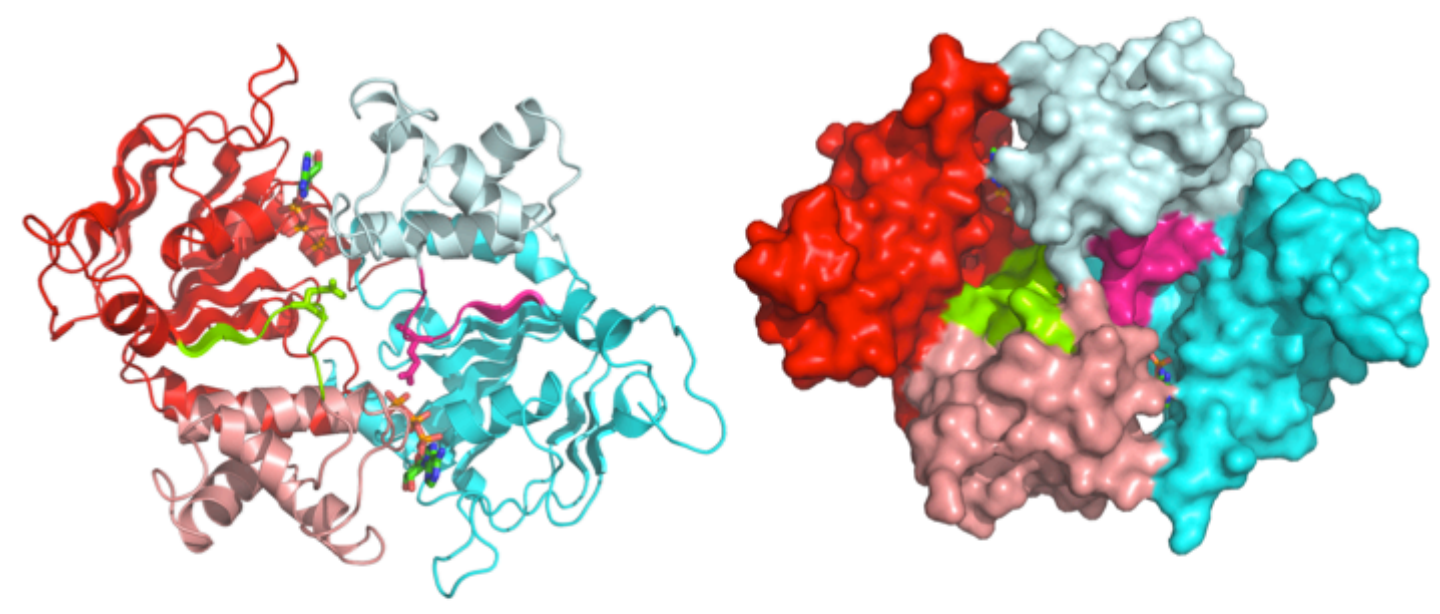
Figure 2

A
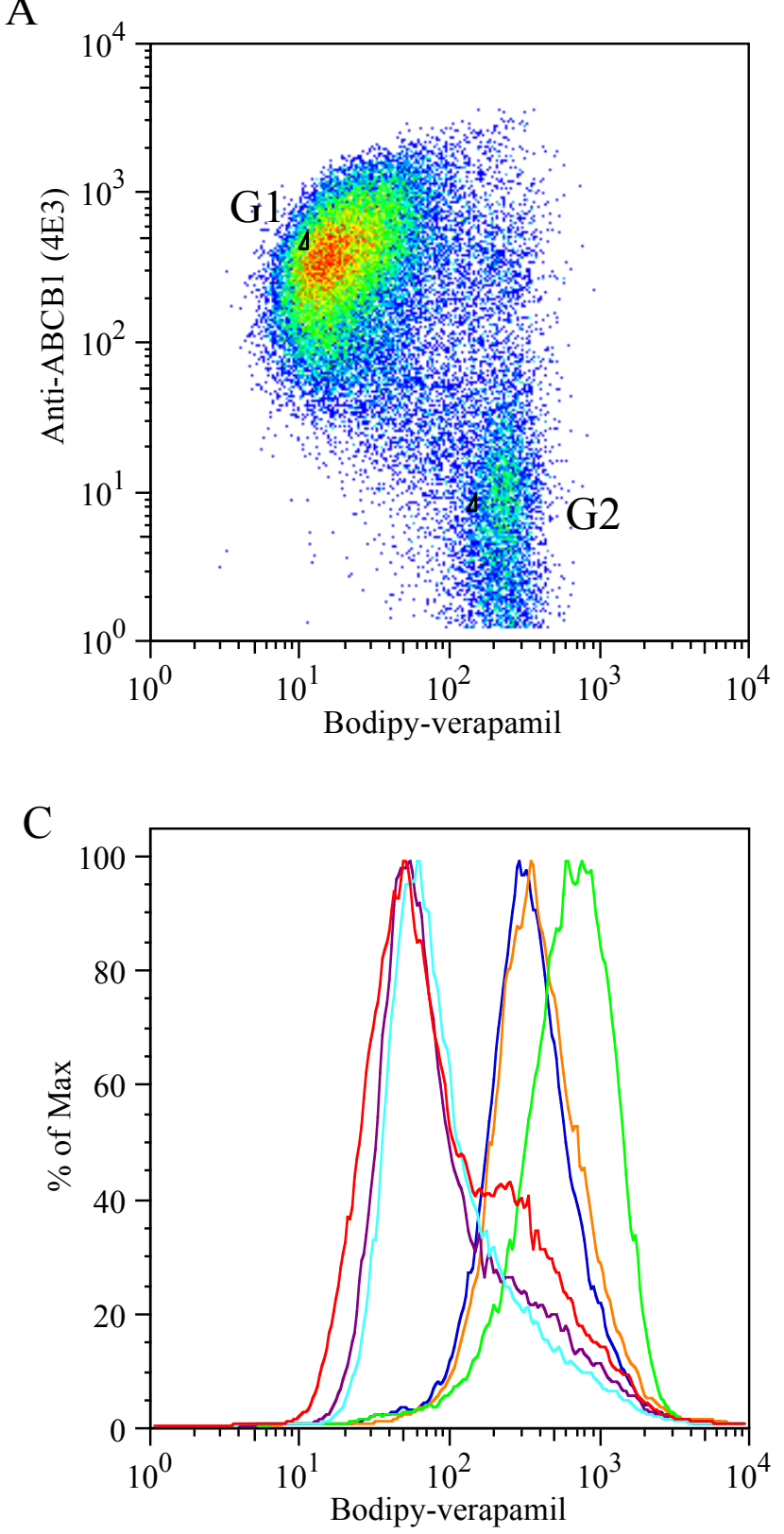

B

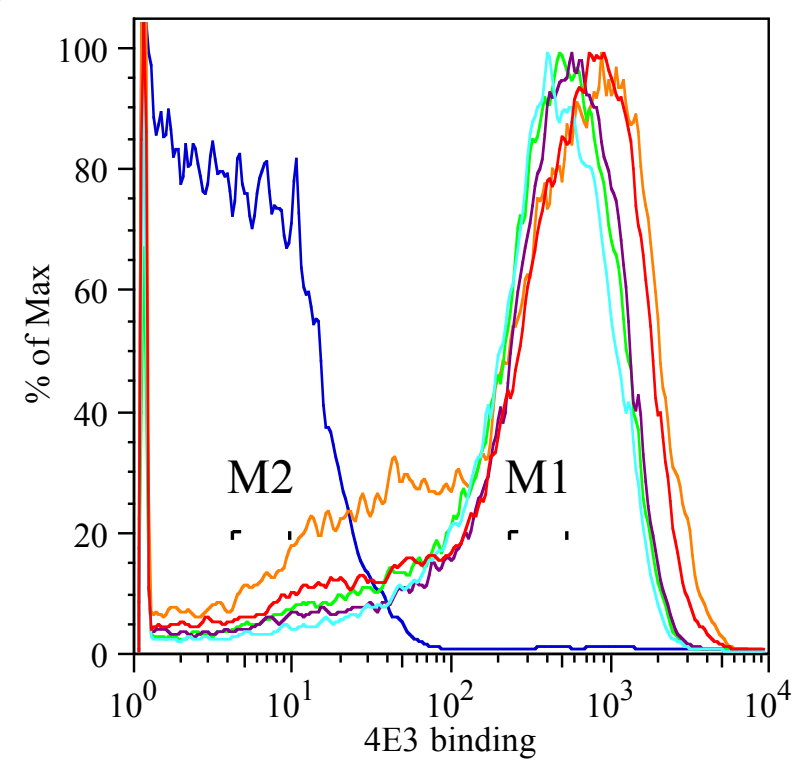

$\square$ wild-type $\mathrm{ABCB} 1$

日 Q475A

日 Q1118A

日 Q475A-Q1118A

日 Walker B E1201Q

日 mock transfected

D

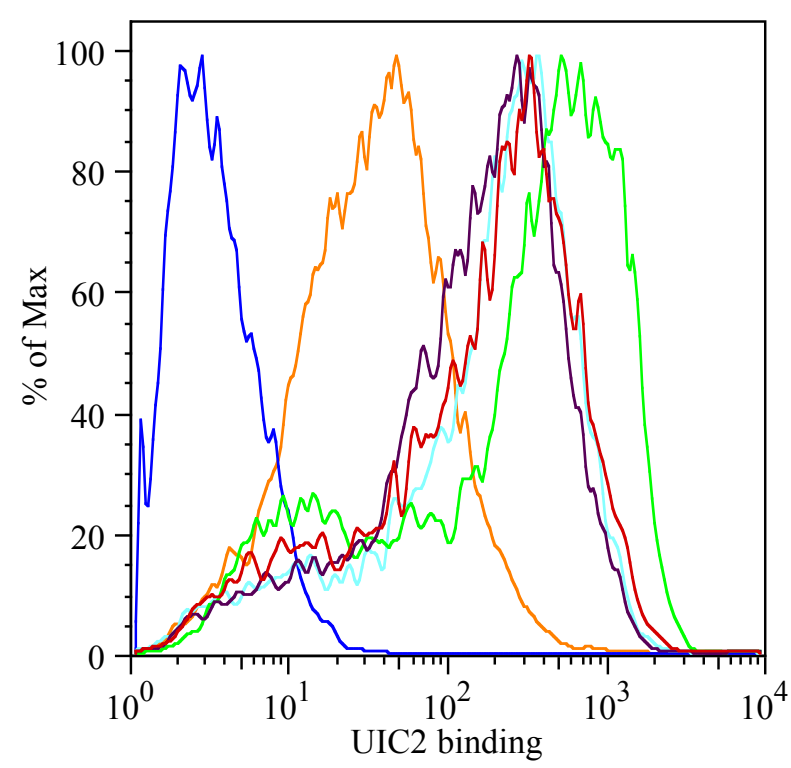


Figure 3.

4E3 Binding

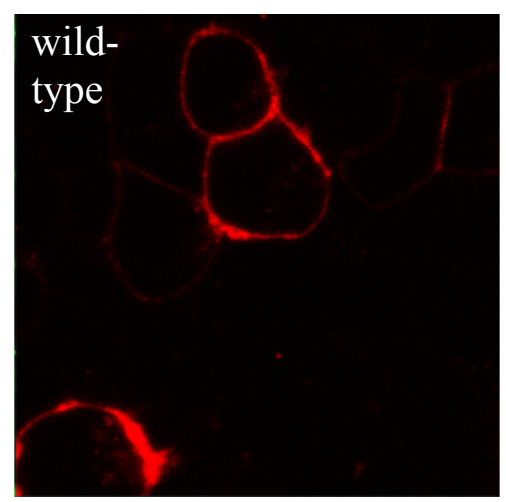

Q475A/ Q1118A
Bodipy-verapamil
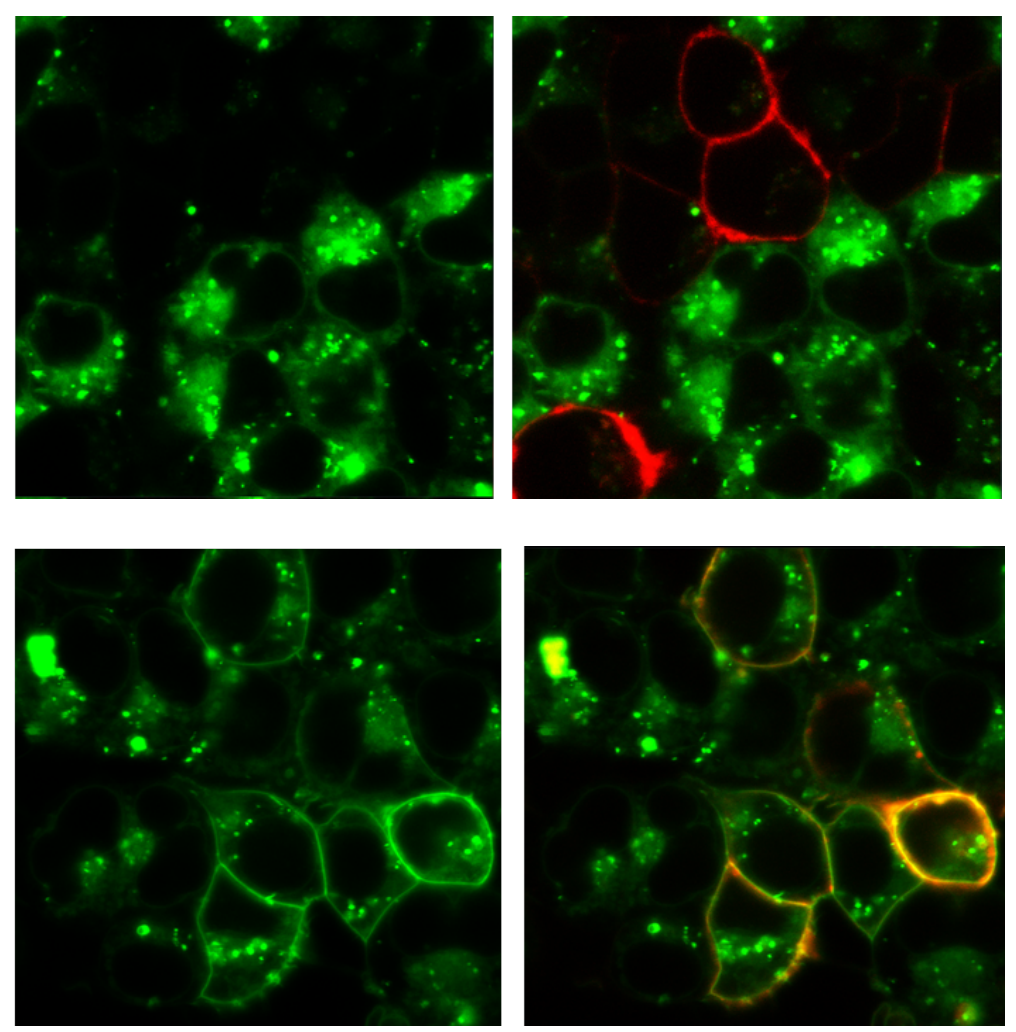

E1201Q
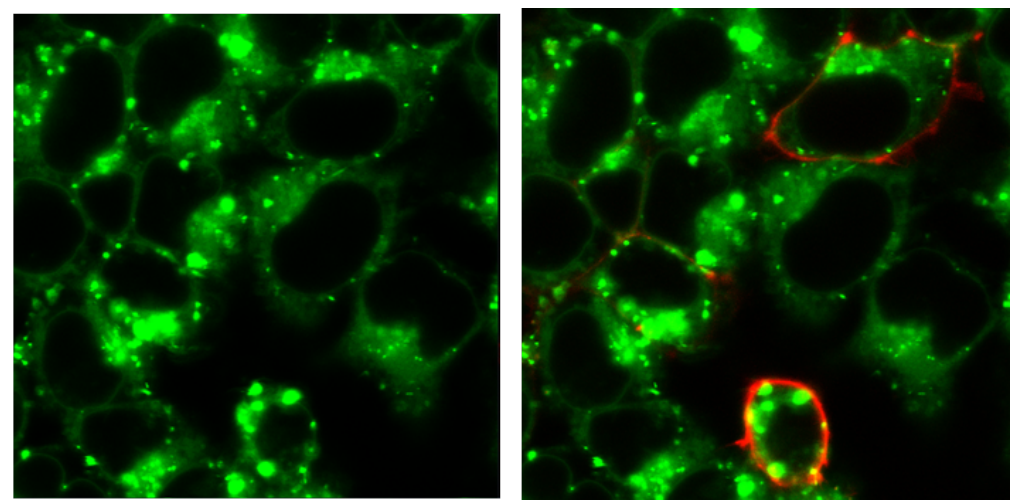
Figure 4

A

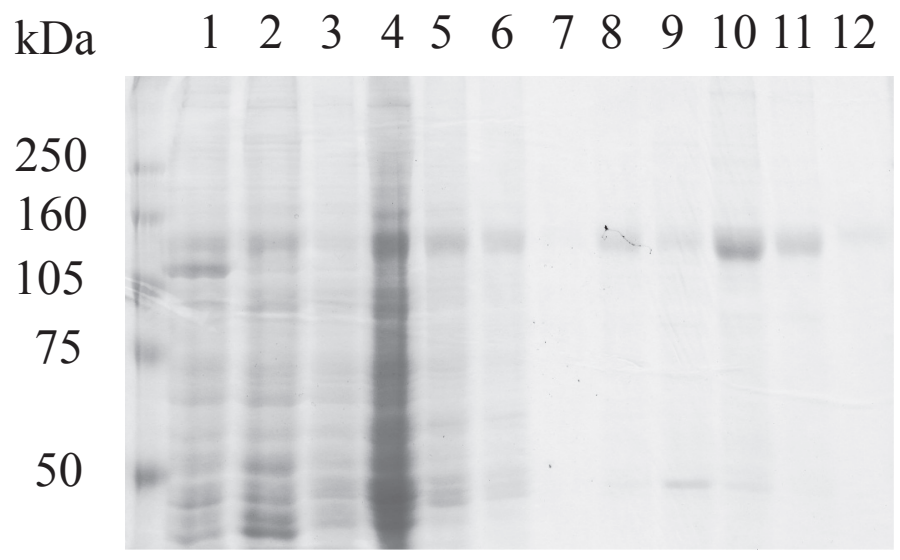

B

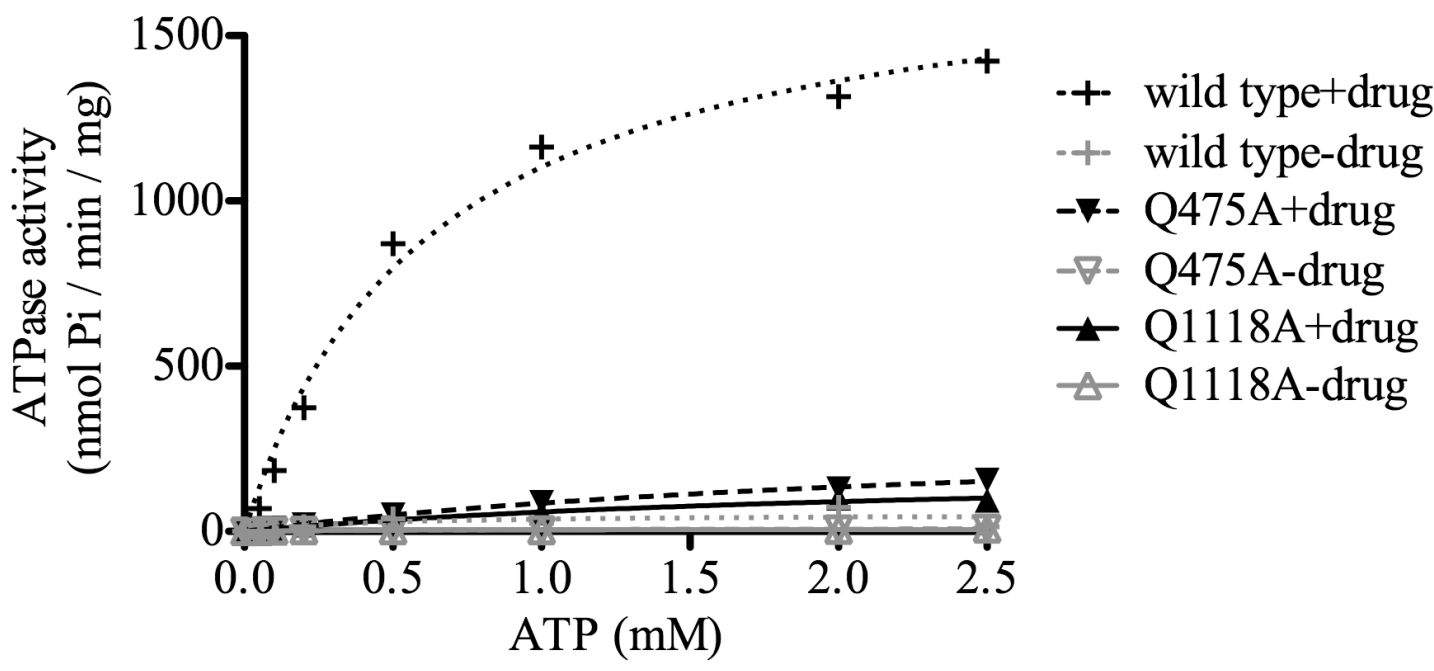

C

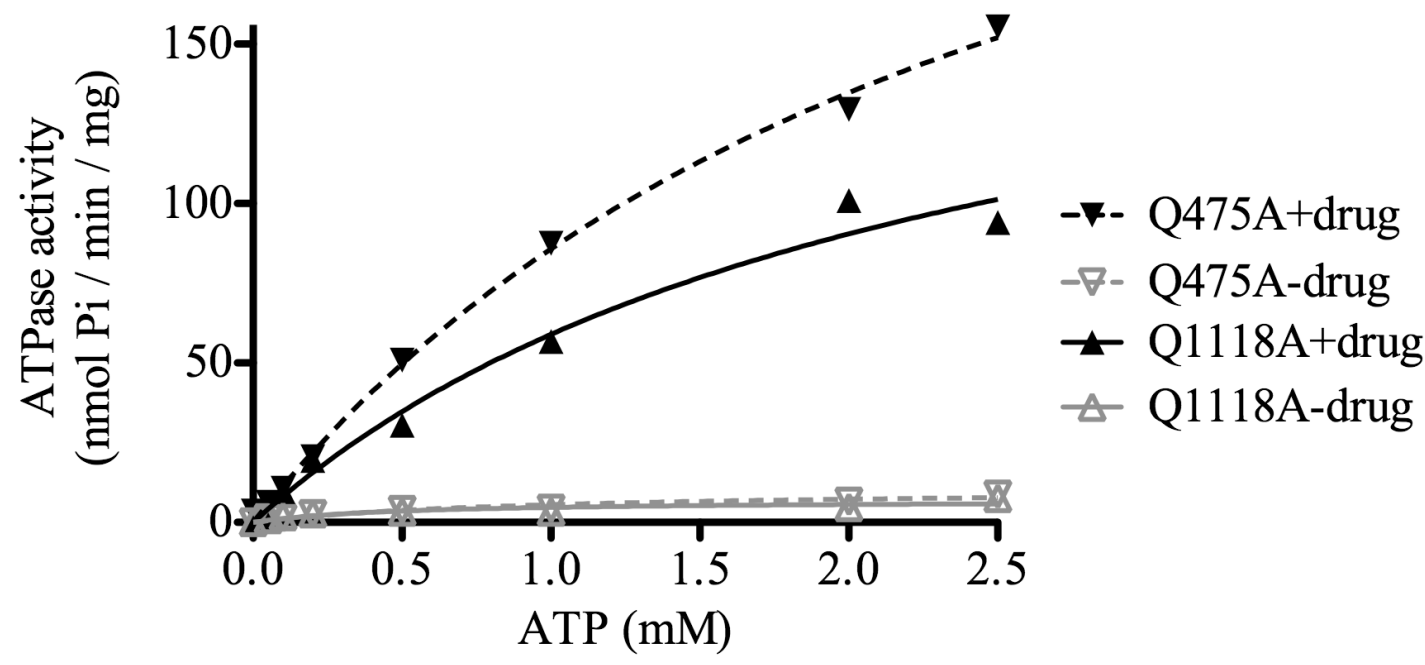


A

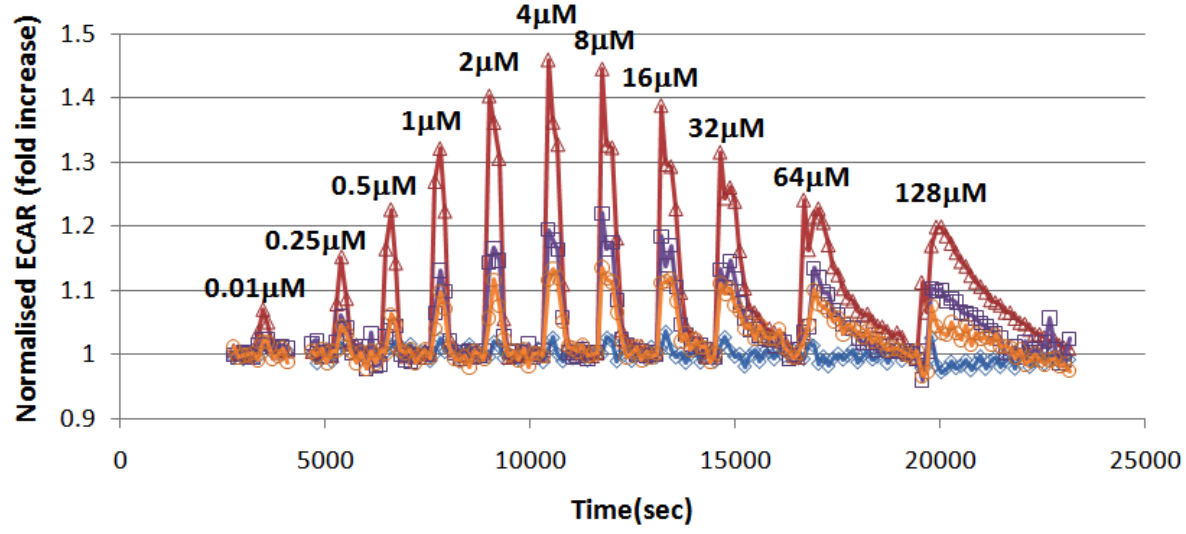

B
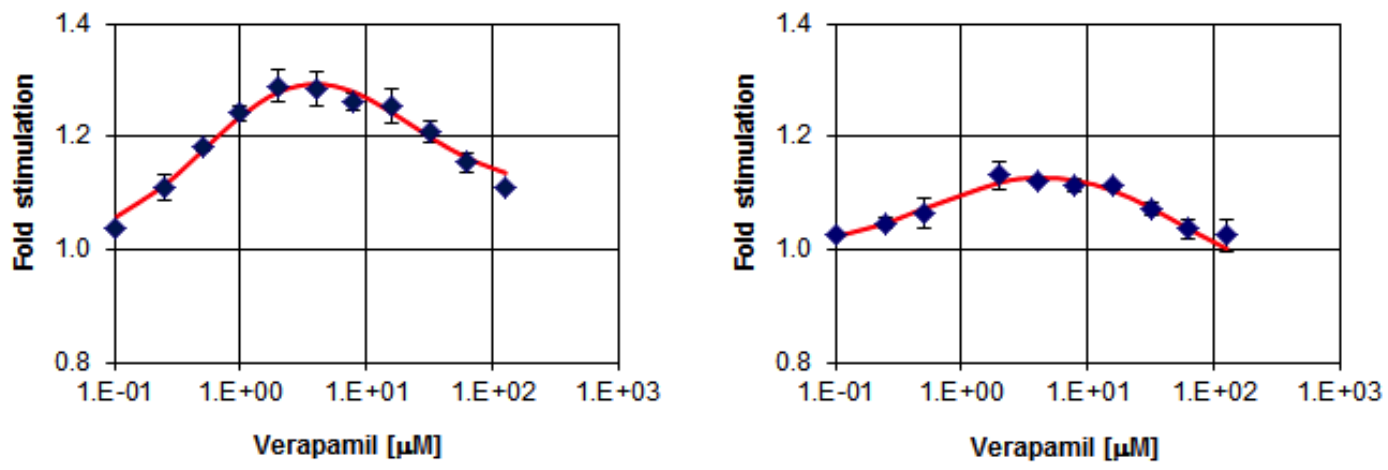

D

$E$
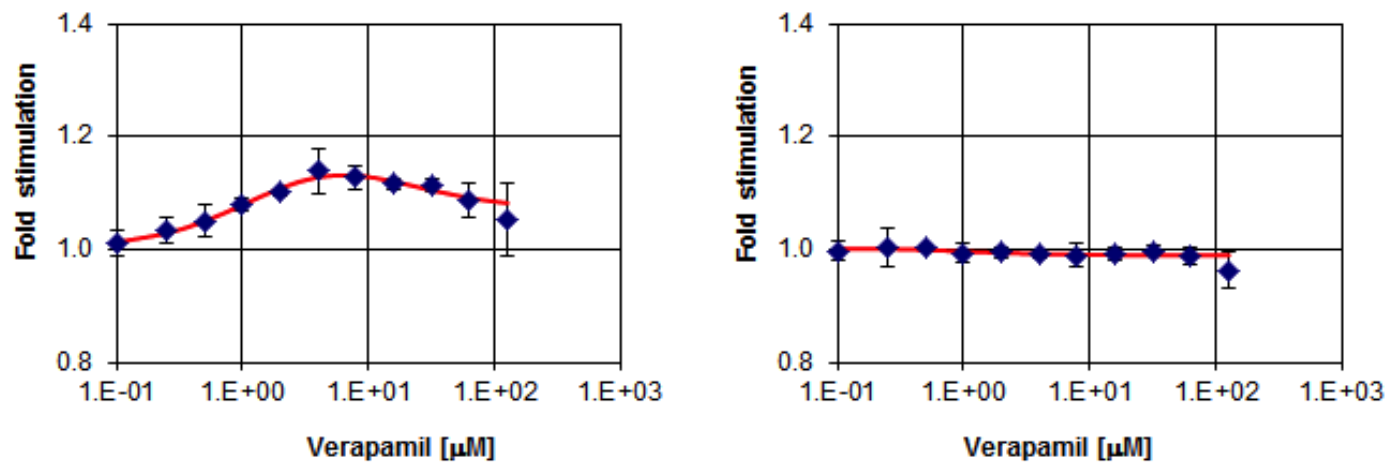
Figure 6

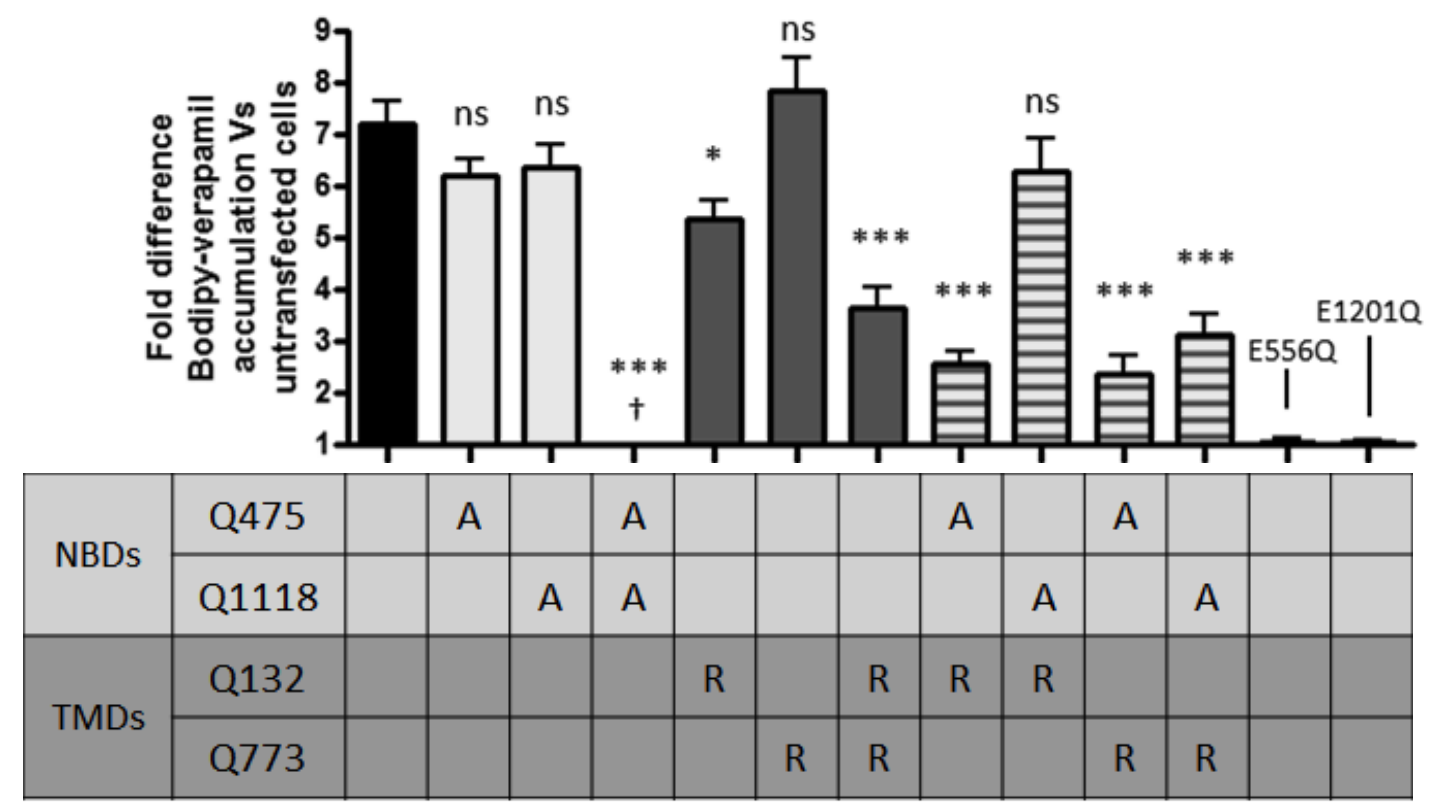



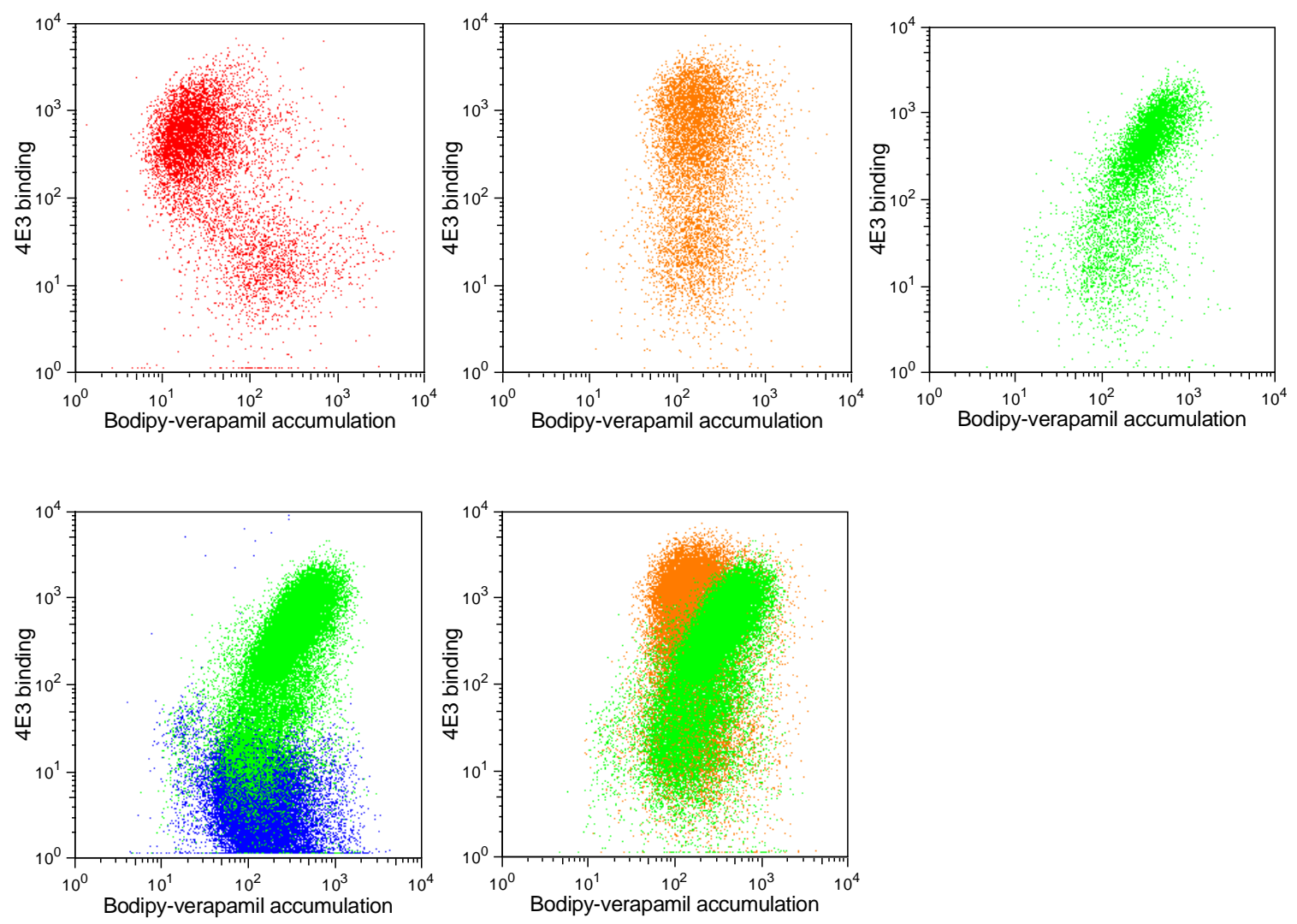

Supplementary Figure 1. The double Q-loop mutant Q475A/Q1118A imports Bodipy-verapamil. Dotplot from two-colour flow cytometry experiment (see methods and materials in the main text for details), showing the relationship between ABCB1 surface expression (4E3 labelling) and Bodipy-verapamil accumulation. Each dot represents an individual cell. In the population of cells expressing wild-type ABCB1 (red, top left panel) increasing surface labelling with 4E3 (and so increasing ABCB1 expression) correlates with decreasing accumulation of Bodipy-verapamil (the population to the bottom right of the plot are non-transfected cells within the population). Cells expressing the catalytically inactive Walker B mutant E1201Q (orange, top centre panel) accumulate Bodipy-verapamil to the same level as mock-transfected (or nonexpressing cells within the E1201Q transfection experiment). In contrast, cells expressing the double Q-loop mutant Q475A/Q1118A (green, top right panel) accumulate more Bodipy verapamil than cells expressing the E1201Q mutant, and the level of accumulation goes up with increasing expression of Q475A/Q1118A. Direct comparison of the level of Bodipy-verapamil accumulation of cells expressing Q475A/Q1118A (green), with mock-transfected cells (blue) and cells expressing E1201Q (orange) is given in the overlay plots to the bottom left and bottom right, respectively. 
A

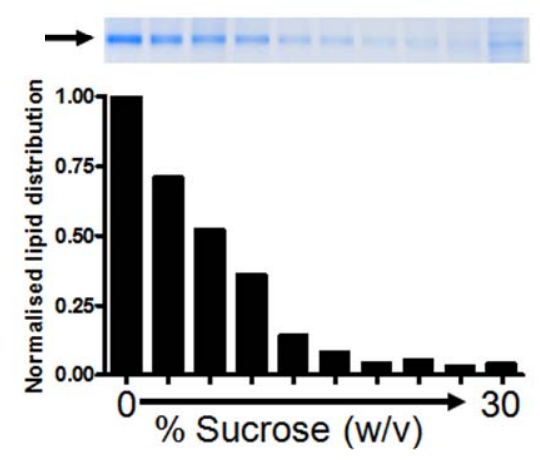

$\overline{\mathrm{C}}$

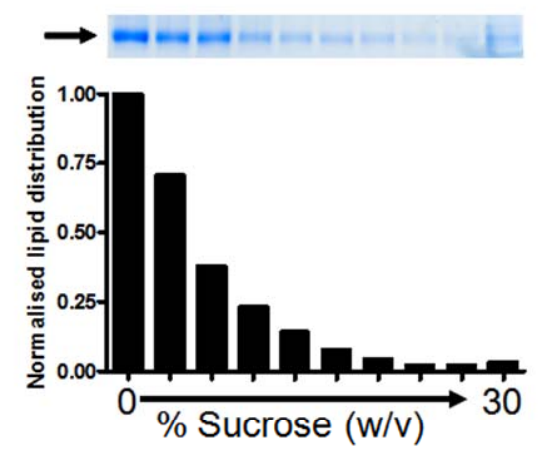

B

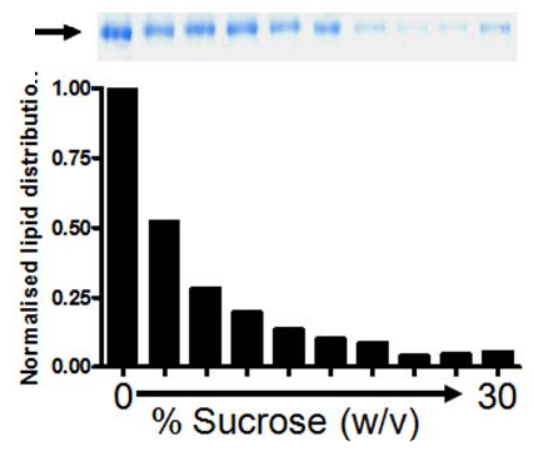

D

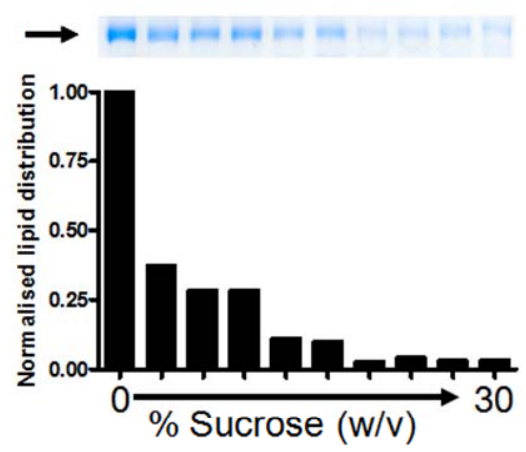

Supplementary Figure 2. Purified wild-type and mutant ABCB1 was reconstituted into liposomes with equal efficiency. Proteoliposomes containing (A) wild-type, (B) NBD1-Q475A, (C) NBD2-Q1118A, and (D) Q475A/Q1118A ABCB1 were separated using a sucrose density gradient (0-30\%). Protein content of each fraction was visualised using SDS-PAGE, followed by colloidal blue staining (top panels, arrows indicate ABCB1). Distribution of ${ }^{3} \mathrm{H}-$ phosphatidylcholine between each fraction was determined using liquid scintillation counting (bar graphs). Co-fractionation of protein and lipid shows efficient incorporation of wild-type and mutant ABCB1 into liposomes. 

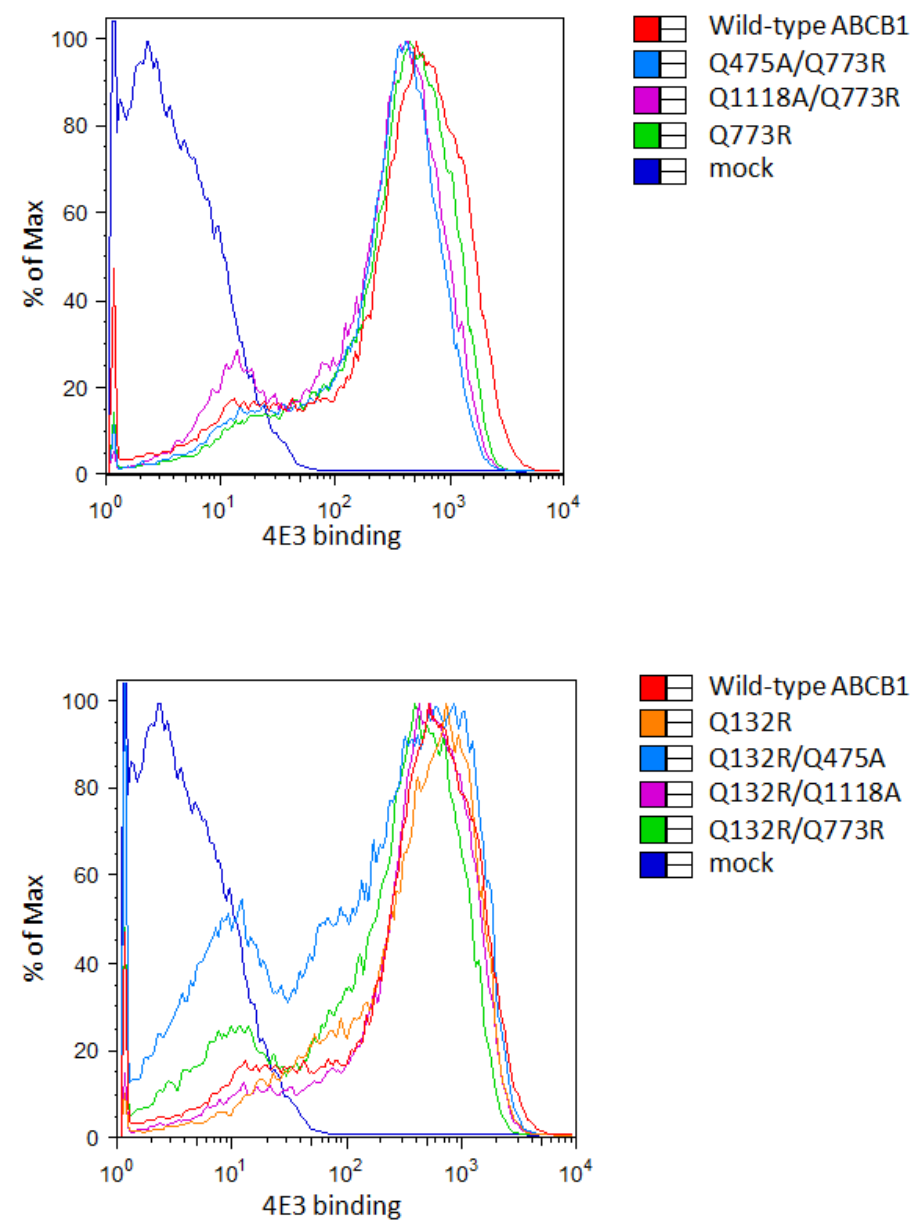

Supplementary Figure 3. TMD mutations Q132R and Q773R do not affect the level of ABCB1 surface expression.

HEK293T cells transiently expressing mutant and wild-type ABCB1 were labelled with saturating concentrations of the ABCB1-specific antibody 4E3 to label total ABCB1 (see materials and methods in the main text for details). 